10

SSCL-Preprint-550

January 1994

Distribution Category; 414

Y. Nosochkov

T. Sen

E. Courant

Principles of Interaction

Region Design in Hadron

Colliders and Their Application to the SSC

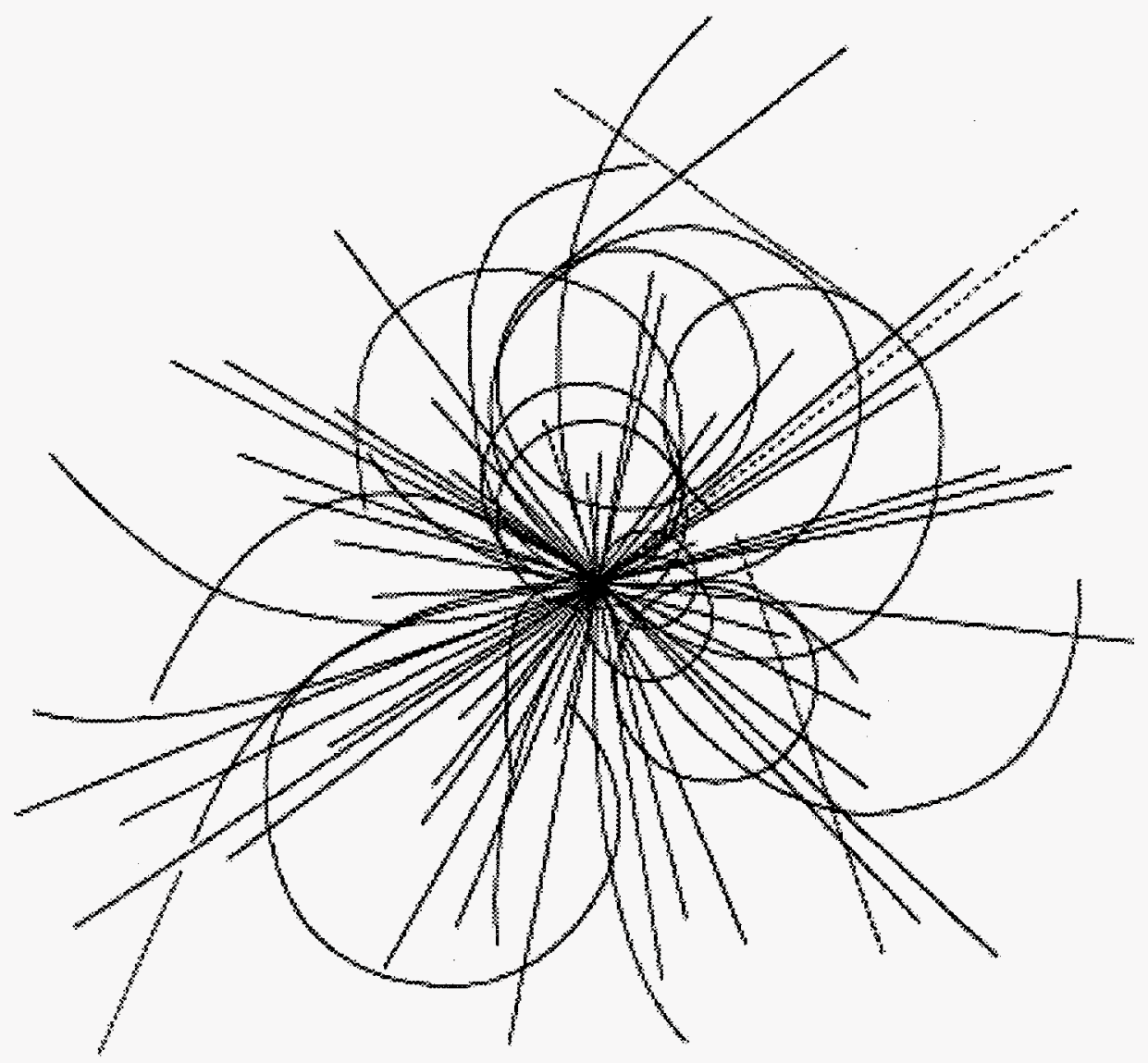

Superconducting Super Collider Laboratory

APP-OUT CN AELEASE OR PUBLICATION - OR. PATENT GROUP

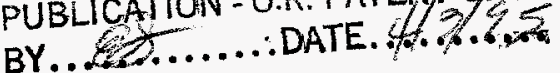




\section{Disclaimer Notice}

This report was prepared as an account of work sponsored by an agency of the United States Govemment. Neither the United States Government or any agency thereof, nor any of their employees, makes any warranty, express or implied, or assumes any legal liability or responsibility for the accuracy, completeness, or usefuiness of any intormation, apparatus, product, or process disclosed, or represents that its use would not infringe privately owned rights. Reference herein to any specitic commercial product, process, or service by trade name, trademark, manufacturer, or otherwise, does not necessarily constitute or imply its endorsement, recommendation, or favoring by the United States Government or any agency thereof. The views and opinions of authors expressed herein do not necessarily state or reflect those of the United States Government or any agency thereot.

Superconducting Super Collider Laboratory is an equal opportunity employer. 


\section{DISCLAIMER}

Portions of this document may be illegible in electronic image products. Images are produced from the best available original document. 


\title{
Principles of Interaction Region Design in Hadron Colliders and Their Application to the SSC*
}

\author{
Y. Nosochkov, T. Sen, E. Courant,$^{\dagger}$ A. Garren, \\ D.M. Ritson,${ }^{\ddagger}$ R. Stiening, and M.J. Syphers \\ Superconducting Super Collider Laboratory $\S$ \\ 2550 Beckleymeade Avenue \\ Dallas, TX 75237
}

January 1994

\footnotetext{
* To be published in Nuclear Instruments and Methods in Physics Research.

$\dagger$ Permanent address: Brookhaven National Laboratory, Upton, NY 11973.

$\ddagger$ Permanent address: Stanford Linear Accelerator Center, Stanford, CA 94309.

$\S$ Operated by the Universities Research Association, Inc., for the U.S. Department of Energy under Contract No. DE-AC35-89ER40486.
} 


\title{
PRINCIPLES OF INTERACTION REGION DESIGN IN HADRON COLLIDERS AND THEIR APPLICATION TO THE SSC
}

\author{
Y. Nosochkov, T. Sen, E. Courant ${ }^{\dagger}$, A. Garren, \\ D.M. Ritson $\neq$, R. Stiening and M.J. Syphers \\ Superconducting Super Collider Laboratory * \\ 2550 Beckleymeade Avenue, Dallas, TX 75237 \\ † Permanent address: Brookhaven National Laboratory, Upton, NY 11973 \\ $\ddagger$ Permanent address: Stanford Linear Accelerator Center, Stanford, CA 94309
}

\begin{abstract}
The high luminosity Interaction Regions (IRs) are an important part of the lattice in colliding beam machines. The performance of the collider may depend significantly on the particular design of the IRs. In this paper we discuss the general principles of IR design and apply these principles to the design of the Superconducting Super Collider Interaction Regions.
\end{abstract}

\section{Introduction}

The requirement of higher luminosities in colliding beam experiments has led to the need for specially designed sections in the lattice of accelerators, called Interaction Regions (IRs). Within an IR, the two beams are brought to collision at an Interaction Point (IP). The layout of the IR must satisfy the specific requirements imposed by the experimental detector on the beam parameters at the IP. The particular design of the IR may vary depending on the type of machine (circular or linear, $p p, p \bar{p}$, $e^{+} e^{-}$, etc.), the beam energy, the space available for an IR, etc. However, the most distinctive requirement for an IR optics is that it has to provide a beam size at the IP substantially different (either lower or higher) from that in the rest of the machine.

*Operated by the Universities Research Association Inc., for the U.S. Department of Energy, under contract DE-AC35-89ER40486. 
Many of the common features and problems in the design of an IR follow from this single requirement. These features and requirements are summarized below:

- A strong final focus quadrupole system (usually triplet or doublet) has to be placed on either side of the Interaction Point to provide a desired beam size at the IP.

- The final focus quadrupoles have to be common to both beams if the beams are to cross at a small or zero angle at the IP, and the value of the amplitude function there, $\beta^{*}$, is low.

- The $\beta$-function attains a very high value $\beta_{\text {peak }}$ in the final focus quadrupoles.

- The final focus quadrupoles must have a bore large enough to ensure a sufficient region of good field quality for the larger beam in these quadrupoles. The orbit displacement due to the crossing angle places even tighter tolerances on the field quality.

- A much lower $\beta_{\text {peak }}$, and thus a different optical configuration, is required at the injection energy where the beam emittance is larger.

- The transition from injection to collision optics, called the $\beta$-squeeze, is accomplished by changing the gradients of a set of tuning quadrupoles.

- Large variations of the $\beta$-function, other than in the final focus quadrupoles, should be avoided.

- In $p p$-colliders the beams are brought into collision by use of a set of dipole magnets. A common dipole with large bore is required on either side of the IP to separate the beams.

- The dispersion must be suppressed at the IP for two reasons: 1) to minimize the transverse beam size and thus maximize the luminosity, and 2) to avoid exciting synchrobetatron resonances that would reduce the beam lifetime [1].

- In all optical configurations the IR must be matched to the adjacent sections in order to avoid perturbation of the lattice functions in the rest of the machine.

- Due to the high $\beta_{p e a k}$, the beam is very sensitive to any errors in the final focus region. This may require special corrections for these quadrupoles, namely nonlinear chromaticity correction, crossing angle correction, and correction of multipole field errors in these quadrupoles. The IR optics should be designed to facilitate these corrections.

Once the $\beta^{*}$ at the IP has been chosen, it is desirable to have the bunch length $\sigma_{l}$ smaller than $\beta^{*}$, to avoid the luminosity reduction due to a significant variation of the transverse size within a bunch at the IP.

In addition to the above, the following considerations should be included in the IR design: 
- The design should be flexible enough to provide a range of sizes for both beams at the IP as well as space for the detector in the event that experimental requirements change. Preferably this should be achieved with only minor modifications of the IR optics.

- The optics should be optimized in order to reduce the different types of magnets and other components in the machine.

Examples of IR design are given, for instance, in References [2] and [3] for the existing proton machines Super Proton Synchrotron (SPS) at CERN and Tevatron at Fermi National Accelerator Laboratory (FNAL), and in [4] for the Large Hadron Collider (LHC), which is being designed at CERN. Earlier versions of the Superconducting Super Collider (SSC) IR optics are described in References [5]-[11]. Similar approaches were used in the above cases to implement the optical features in the IR. One observes that problems in the IR design are usually caused by allocation of insufficient space for the IR or by additional constraints on the geometry of this section. It is therefore important that the geometrical layout of the IR be determined at an early stage in the design of the lattice.

Our study of the Interaction Regions at the SSC has led to an improvement in the optical properties of the IR and an increased flexibility of the optics. The present design is also more stable with respect to the field errors in the final focus quadrupoles, the major sources of perturbation in the IR. Special optical features have been included into the IR lattice in order to meet the above requirements. The basic features of the design are:

- The IR optics is antisymmetric about the IP in order to provide identical focusing properties for both proton beams. Note that one beam, coming into the IR from one side, experiences the same sequence of focusing strengths as does the other beam coming from the opposite side.

- Each half IR is divided into two major sections: 1 ) the $2 \pi$ final focus section, and 2) the tuning section. This provides a two-step focusing of the beam at the IP and allows almost independent adjustment of each section.

- The $2 \pi$ phase advance final focus section covers the region from the IP to a secondary focal point (SF). It includes the final focus triplet quadrupoles and a region called the $M=-I$ section. The latter is an insertion that does not affect the $\beta^{*}$ value, but cancels the vertical dispersion at the IP. The whole section provides point-to-point focusing from SF to IP with a fixed transfer matrix and $2 \pi$ phase advance. It is important that the $(1,2)$ coefficient of each $2 \times 2$ transfer matrix be zero for two reasons: 1 ) the phase advance across this section does not depend on the $\beta^{*}$, and 2) there is a constant linear transformation of $\beta$-function from the SF to the IP.

- The optics of the $2 \pi$ section acts on the beam as a constant magnification telescope producing an image at the SF of the beam at the IP. The SF may be 
useful for beam diagnostics, and protection collimators may be placed here to intercept particles that might otherwise strike the sensitive inner regions of the detector.

- The final focus quadrupoles have constant gradients during the $\beta$-squeeze. The advantages of this are: 1) the squeeze can be done independently for each ring, and 2) additional eddy current field errors in these quadrupoles are not introduced during the squeeze.

- There is enough free space between the IP and SF to allow the total space for the detector to vary from $41 \mathrm{~m}$ to $180 \mathrm{~m}$.

- The tuning section includes variable gradient quadrupoles to provide the $\beta$-squeeze from injection to collision optics. It has a variable transfer matrix with fixed phase advance in any optical configuration. The latter is to keep constant (and optimum) the phase advance across half an IR and, hence, the phase advance from the IP to the local IR correctors located in the regions outside the IR.

- Local chromaticity correction and crossing angle systems were assumed for the IR, with sextupole and quadrupole correctors located in the adjacent regular cells in the arcs. These correctors mostly compensate the effect of errors in the final focus quadrupoles. To optimize these corrections a specific value of phase advance across each half IR has been chosen.

- An optimum, fixed phase advance has been chosen between the two IPs in each cluster in order to compensate the second-order chromatic effects produced by the high $\beta_{\text {peak }}$ final focus quadrupoles in the two IRs.

Below we review the details of our solution, a shorter description of which can be found in References [12], [13].

\section{IR Configuration}

There are four Interaction Regions in the SSC proton-proton Collider, located in pairs in the East and West clusters, respectively [11]. The goal for the East IRs is to provide a high value of the luminosity to a maximum of $10^{33} \mathrm{~cm}^{-2} \mathrm{~s}^{-1}$ and, hence, a low $\beta^{*}$ at the IP. In the West IRs significantly larger space is required for the detectors. This is achieved at the cost of a higher value of $\beta^{*}$ and correspondingly lower luminosity.

Figure 1 shows a vertical schematic view of a complete IR. Its total length is $1890 \mathrm{~m}$. The first beam goes along its beam line from the top left side of the picture to the bottom right, while the second beam starts at the top right and proceeds to the bottom left. They cross each other at the IP in the center of the IR. There are two IRs in the cluster separated by a short section with horizontal bending. Therefore, 
the beams interchange their vertical positions twice while passing through both IRs and return to their initial vertical positions at the exit of the second IR.

In Figure 1 the dipoles straddle the beam lines, the focusing quadrupoles are shown above the lines, and the defocusing quadrupoles below. In each ring, the optics is antisymmetric with respect to the IP. This means that the magnet locations have a mirror symmetry about the IP, but the field polarities are opposite for the corresponding magnets on the left and right sides of the IR. The optics is identical in both rings except that corresponding top and bottom quadrupoles have opposite focusing properties and corresponding vertical bending dipoles bend the beams in opposite directions. Each half of an IR is composed of the two major modules specified in the previous section. Taking into consideration the dispersion cancellation property, we can extend the number of the modules to three, which are located geometrically in different regions. Figure 2 shows the layout (not to scale) of half of an IR. The three modules are:

- The final focus triplet located next to the IP and common to both rings.

- The $M=-I$ section placed in the region with a vertical separation of $45 \mathrm{~cm}$ between the top and bottom rings.

- The tuning section located in the region of normal vertical separation of $90 \mathrm{~cm}$ between the rings in the Collider.

Between the focusing modules there are vertical dipoles placed in two steps, which bring the beams into collision at the IP.

\section{Optical Modules}

\subsection{Final Focus Triplet}

There are four quadrupoles in the triplet, which form a F3-D2-D2-F1 optical structure for a beam approaching the IP and a D1-F2-F2-D3 structure for a beam leaving the IP. This combination is called a triplet because the two central quadrupoles are essentially one long quadrupole split into two for convenience of fabrication of these magnets. The separation of the quadrupoles in the triplet is very small compared to their focal length. This arrangement preserves the roundness of the beams better than a focusing doublet would, and provides a lower $\beta_{\text {peak }}$ value as well. These quadrupoles and adjacent splitting dipoles BV1c are common to both rings. The proton beams, therefore, share the same beam pipe inside these magnets and, because of the opposing beam directions, they experience opposite magnetic strengths in each triplet magnet. With this constraint, the only way to have an identical lattice for the IR in both rings is to require optical antisymmetry of the whole Interaction Region. The triplet quadrupole polarities shown in Figure 1 correspond to the beam going from the top left side to the bottom right. Opposite polarities are implied for the second beam. 
The luminosity at the collision point depends on the number of protons per bunch $N_{B}$, the bunch spacing $S_{B}$, the beam emittance $\epsilon$ (unnormalized), and the value of $\beta^{*}$ at the IP. For head-on collisions of equal round beams, the expression for the luminosity is

$$
\mathcal{L}=\frac{N_{B}^{2} c}{4 \pi \beta^{*} \epsilon S_{B}} .
$$

To achieve a high luminosity at collision, the beam cross section must therefore be squeezed to a very small size at the IP. This requires quite strong (i.e., long) triplet quadrupoles. The small beam size at the IP leads to a very large divergence of the beam throughout the region reserved for the detectors. The beam size therefore quickly increases with the distance from the IP and reaches an extremely large value in the triplet. For instance, in our solution with $\beta^{*}=0.5 \mathrm{~m}$ and $L^{*}=20.5 \mathrm{~m}$, the $\beta_{\text {peak }}$ in the triplet at collision is about 30 times larger than that in the arcs, where $L^{*}$ denotes the distance from the IP to the first of the final focus quadrupoles. In turn, the beam divergence in the triplet becomes very small. As a result the beam becomes much more sensitive to the field errors in the triplet than in any other region of the machine. This occurs for the following reasons:

- The larger beam in the triplet samples regions of higher multipole field errors. This effect is amplified by large orbit displacements due to the crossing angle at the IP.

- Any kick due to field errors results in a larger perturbation of the betatron motion because of the small beam divergence in the triplet.

- Large integrated gradient over the length of the triplet.

The first two points are a consequence of the very large $\beta$-function values in the triplet. The scale of the effect of the triplets can be easily seen in the example of linear chromaticity at collision conditions. The linear chromaticity due to two triplets ( 8 quadrupoles) in one IR at $\beta^{*}=0.5 \mathrm{~m}$ is about $31 \%$ of that due to the 784 quadrupoles in the Collider arcs. Roughly, the effects of field errors in the final focus triplets are one to two orders of magnitude larger than those generated by errors in quadrupoles placed in the arcs. The effects of errors in the triplets will be discussed in more detail in Section 8.

In the IR optics of other machines (see, for instance, References [2], [3], [4]) the gradients of final focus quadrupoles are changed during the $\beta$-squeeze along with the gradients of the other tuning quadrupoles in the IR. In fact, the variation of the gradients in the triplets is very small, because the beam is specially sensitive to field changes in this region. For reasons mentioned in Section 1, we keep the triplets at constant gradients during the squeeze. Since the constraints require six tuning quadrupoles, freezing the three in the triplet requires three additional tunable quadrupoles.

The accommodation of three extra quadrupoles in the IR requires an additional straight section region within the IR. Historically, this extra length was introduced 
at first to allow later addition of a bypass section containing two extra IRs radially inward from the original outer IR pair. This combination of four IRs forms the socalled diamond bypass structure, which is described in Reference [11]. In this paper we do not describe the lattice of the inner bypass, assuming this to be a future upgrade of the lattice.

In $p p$-colliders with multi-bunch beams, a non-zero crossing angle is introduced at the IP in order to avoid the large number of head-on collision points in the experimental region, and to reduce the detrimental effects of long-range beam-beam interactions. This results in large orbit displacements in the final focus quadrupoles, which are up to $5 \mathrm{~mm}$ in our case [14]. The nonlinear fields in these quadrupoles can then significantly affect long-term stability. Studies of the dynamic aperture [15] have shown that it is more important to have a large bore in the triplet than to reduce the beam size by increasing the gradient. For the IR optics with the maximum $\beta_{\text {peak }} \approx 9 \mathrm{~km}$ our choice is a bore of $5 \mathrm{~cm}$ for the triplet quadrupoles with a maximum gradient of about $191 \mathrm{~T} / \mathrm{m}$. This choice was based on cost rather than performance: 5 -cm quadrupoles had been developed for other uses in the SSC Collider. Studies showed that quadrupole bores between 6 and $7 \mathrm{~cm}$ would have provided superior performance [15].

\section{2 $M=-I$ Section}

The two beams circulating in different rings are brought into collision at the IP by use of a set of vertical dipoles. These dipoles, in turn, create a vertical dispersion $\eta_{y}$ which, if not corrected, would propagate through the whole machine. For instance, the effect of uncorrected dispersion in one $I R$ alone results in about $2.5 \mathrm{~m}$ of residual vertical dispersion in the Collider arcs at collision configuration, and this grows to $6.4 \mathrm{~m}$ when dipoles from four identical low- $\beta$ IRs are included.

To correct the vertical dispersion, the scheme of a two-step orbit change has been used for each half of an IR with a $M=-I$ section between the steps. Optically, the $M=-I$ section is composed of two identical FODO cells with double quadrupoles and a phase advance of $90^{\circ}$ per cell. The transfer matrix across this section is, therefore, the negative identity matrix, hence the name. The inclusion of this section does not disturb the $\beta$-functions in the regions outside, but it does change the betatron tune by a half unit per section.

The principal idea of dispersion cancellation is illustrated in Figure 3. There are two pairs of vertical dipoles on either side of the $M=-I$ section. The first pair (BV2+, BV2-), of equal strengths and opposite polarities, changes the orbit of one beam by $h_{1}=22.5 \mathrm{~cm}$. The second pair, similarly arranged on the other side of the $M=-I$ section, produces the same step $h_{2}=22.5 \mathrm{~cm}$. The dispersion generated by each pair of dipoles follows the change of the orbit; hence, at the exit of the first pair it is equal to $\eta_{y 1}=h_{1}, \eta_{y 1}^{\prime}=0$. The slope of the dispersion at the exit being zero, the dispersion at the entrance of the $M=-I$ section is the same no matter how far it is from the dipoles. In the $M=-I$ section the dispersion follows free betatron motion and changes sign at the exit of this section: $\eta_{y 2}=-\eta_{y 1}=-h_{1}$, 
$\eta_{y 2}^{\prime}=-\eta_{y 1}^{\prime}=0$. It propagates unchanged up to the next dipole, then the second pair of dipoles (BV1+, BV1c-) deflects the orbit by $h_{2}$ back toward the center, resulting in a complete cancellation: $\eta_{y}=\eta_{y 2}+h_{2}=-h_{1}+h_{2}=0, \eta_{y}^{\prime}=0$.

The above scheme is a special case of a more general configuration with unequal steps $h_{1} \neq h_{2}$, in which the dispersion could be corrected using the following transformation instead of $M=-I$ :

$$
M=\left[\begin{array}{cc}
-h_{2} / h_{1} & 0 \\
0 & -h_{1} / h_{2}
\end{array}\right]
$$

This section compensates any orbit perturbation due to systematic field errors in the vertical bend dipoles the same way it does the vertical dispersion. The important advantages of such a correction scheme are that it provides a local compensation of the dispersion, and it does not depend on the $\beta^{*}$.

The only reason for including the $M=-I$ section is to cancel the dispersion. Therefore, this section is made very short in order to reserve additional space for larger $L^{*}$ options of the IR. Its total length is about $150 \mathrm{~m}$, which is significantly smaller than $360 \mathrm{~m}$, the length of two standard arc cells producing the same phase advance of $180^{\circ}$. Stronger quadrupoles are needed to provide the same focusing in a shorter distance; hence, double quadrupoles are used in this section. Compared to a FOFDOD option of the $M=-I$ section, the FODO scheme reduces the total quadrupole length at the expense of a higher beta peak $(1.3 \mathrm{~km})$ in this region at collision. This is, however, much smaller than the main peak of $9.1 \mathrm{~km}$ in the final focus triplets.

It is clear from the above consideration that the $M=-I$ section can be placed anywhere between the two pairs of dipoles without perturbing the optics. In order to reduce the beta peak in this region and the IR chromaticity at collision, as well as to provide the most space for the detector, this section was moved as far as possible from the IP.

Geometrically the $M=-I$ section and adjacent vertical dipoles are located in the region with a separation of $45 \mathrm{~cm}$. The small distance between the beams in this region requires a special 2-in-1 magnet design for this section. The technical design and fabrication of this section are expected to be more complicated than those for the standard magnets.

A configuration without the $M=-I$ section has been studied [13], [16]. It could significantly reduce the total cost of the IR magnets and provide additional available space for the detector. In this case, however, compensating the vertical dispersion becomes quite complicated. The dispersion can be locally corrected by use of a set of skew quadrupoles located in the regions with dispersion, but the required strengths of these correctors significantly increase in the low- $\beta$ IR configurations. One possibility is to use such a scheme in a high- $\beta$ option of the IR design. 


\subsection{Secondary Focus}

Another new feature of the present IR design is an inclusion of a secondary focal point. This point separates each half of an IR into two regions: 1) a tuning section with independent variable gradients, and thus with a variable transfer matrix, and 2) a central region (with constant transfer matrix and $2 \pi$ phase advance) from the IP to $\mathrm{SF}$, which includes the final focus triplet and the $M=-I$ section. It is desirable that the phase advance across the constant transformation section be an integral multiple of $\pi$. In this case the optics of this section provides point-to-point focusing from IP to SF. It acts on the beam as a telescope and creates at the SF a magnified image of the beam at the IP. The advantages are that 1) the phase advance between the IP and $\mathrm{SF}$ does not depend on the $\beta^{*}$, and 2) there is a constant linear transformation of the $\beta$-function and beam size from the IP to the SF.

It follows from the optical symmetry that a SF exists in each half of an IR next to the outermost BV2 dipoles at a distance of $\approx 541 \mathrm{~m}$ from the IP (see Figure 2).

The transfer matrix between the IP and the SF is determined by the three strengths of the triplet quadrupoles and the length of this region. The distance from the IP to the triplet is not to be considered as a free parameter here, as it depends mostly on experimental requirements. The lengths of two triplet quadrupoles are adjusted to create a phase advance of $2 \pi$ between an IP and the SF in both planes. The length of the third quadrupole (the one nearest to the IP) is adjusted to minimize and, thus, to equalize the horizontal and vertical beta peaks in the triplet at collision. The transfer matrix from the secondary focus to the IP is, therefore, of the following form:

$$
M=\left[\begin{array}{cc}
\sqrt{\frac{\beta^{*}}{\beta_{s f}}} & 0 \\
\frac{\alpha_{s f}}{\sqrt{\beta^{*} \beta_{s f}}} & \sqrt{\frac{\beta_{s f}}{\beta^{*}}}
\end{array}\right],
$$

where the subscript $s f$ denotes the betatron functions at the SF. There are no tuning quadrupoles between the secondary focus and the IP; hence, the coefficients of the above matrix are constant. This means a phase advance of $2 \pi$ across this region for any initial $\beta$-functions, and a constant magnification of the beam size from the IP to the SF:

$$
m_{s f}=\sqrt{\beta_{s f} / \beta^{*}} .
$$

For the low- $\beta$ IR with $L^{*}=20.5 \mathrm{~m}$, the magnification $m_{s f}$ in the two planes is 4.6 and 7.5, respectively. The $x$ and $y$ magnifications are exchanged on opposite sides of the IP due to the optical antisymmetry.

There are not enough controls to ensure that the $\alpha$-function at a secondary focus is zero. The minimum of the $\beta$ location or the waist in the beam cross section is shifted away from the SF. The shift in a field-free region equals

$$
\Delta s=\frac{\alpha_{s f} \beta_{s f}}{1+\alpha_{s f}{ }^{2}} .
$$


In this case $\beta_{s f}$ differs from $\beta_{\min }$ by the following amount:

$$
\frac{\beta_{s f}-\beta_{\min }}{\beta_{s f}}=\frac{\alpha_{s f}^{2}}{1+\alpha_{s f}^{2}} .
$$

It is seen from Equation (3) that $\alpha_{s f}$ is proportional to $\beta^{*}$ :

$$
\alpha_{s f} \propto \sqrt{\beta^{*} \beta_{s f}}=m_{s f} \beta^{*}
$$

Therefore, at collision conditions with low $\beta^{*}, \alpha_{s f}$ is small and the location of $\beta_{\text {min }}$ is close to the SF. For the $\beta^{*}=0.5 \mathrm{~m}$, the above shift $\Delta s$ is $(0.3 \mathrm{~m}, 2.7 \mathrm{~m})$ in the two planes, and the difference between $\beta_{s f}$ and $\beta_{\min }$ is less than $1 \%$. At injection, the waist in one plane is $55 \mathrm{~m}$ from the $\mathrm{SF}$, while in the other plane it moves into the tuning section and effectively disappears.

\subsection{Tuning Section}

The tuning section covers the region from the beginning of the IR to the secondary focus. Its length is about $404 \mathrm{~m}$. All the magnets in this region are separated vertically in the two rings by $90 \mathrm{~cm}$, the same as in the rest of the Collider. The main purpose of this section is to bring about a smooth transition of the $\beta^{*}$, called a $\beta$-squeeze, from an injection value of $7 \mathrm{~m}$ to $0.5 \mathrm{~m}$ at collision conditions. This is done by varying six independent currents in the tuning quadrupoles QL4, QL5,..., QL9. Six controls are required to meet the following conditions:

- A constant matched transfer matrix across the full IR during the transition.

- A specific value of the $\beta$-function at the IP.

- Zero slope of the $\beta$-function at the IP.

- Specific values of phase advance across each half IR.

The above conditions are satisfied by changing the transfer matrix across the tuning section, but keeping the transfer matrix from the SF to the IP constant. In particular, $\beta_{s f}$ varies so that the $\beta$-function at the IP obeys: $\beta^{*}=\beta_{s f} / m_{s f}{ }^{2}$.

The locations and lengths of the tuning quadrupoles have been adjusted in order to minimize the following:

- The peak values of $\beta$-functions in this region for a full range of tuning currents.

- The range of the tuning currents during the $\beta$-squeeze.

- The total length of all tuning quadrupoles.

- The number of quadrupole families with different lengths. 


\section{Optical Properties}

A primary goal is to design an IR configuration flexible enough to accommodate a range of achievable luminosities and detector configurations. Optically this requires that the design be compatible with a range of values for both $\beta^{*}$ and the free space reserved for the detector. In this field-free region the $\beta$-function changes quadratically with the distance $s$ from the IP:

$$
\beta(s)=\beta^{*}+\frac{s^{2}}{\beta^{*}} .
$$

A lower value of $\beta^{*}$ results in a larger beam divergence within the detector and, thus, in a larger $\beta_{\text {peak }}$ in the triplets. The maximum allowed value of the $\beta_{\text {peak }}$ determines the lowest feasible value of $\beta^{*}$ and, thus, the maximum luminosity. The constraint that $\beta_{\text {peak }}$ stays constant in all configurations requires that $\beta^{*}$ increases as $L^{*}$ increases.

A consequence of the low $\beta^{*}$ and high $\beta_{\text {peak }}$ values is that the phase advance between the triplet and the IP is very close to $\pi / 2$ and it is practically unchanged across the triplet:

$$
\mu_{I P}-\mu_{t r p l}=\int_{0}^{L^{*}} \frac{d s}{\beta^{*}+s^{2} / \beta^{*}}=\arctan \frac{L^{*}}{\beta^{*}} \approx \frac{\pi}{2},
$$

for $L^{*} / \beta^{*} \gg 1$. Because the phase advance is nearly the same for all quadrupoles in the triplet, the effect of their field errors is equivalent to the effect of a point source located $\pi / 2$ in phase from the IP. The correction magnets, therefore, can be placed at optimum phase positions with respect to all triplet quadrupoles simultaneously, and can compensate their errors most effectively.

For a high- $\beta^{*}$ IR configuration $\left(L^{*} / \beta^{*} \ll 1\right)$, the phase advance between the triplet and an IP is very small; hence, both triplets have almost the same phase advance and their field errors act coherently.

In our solution, the quadrupoles with variable gradient can be tuned to a minimum $\beta^{*}=0.25 \mathrm{~m}$ for the IR configuration with $L^{*}=20.5 \mathrm{~m}$. However the larger value of $\beta^{*}=0.5 \mathrm{~m}$ is sufficient to achieve the design luminosity of $10^{33} \mathrm{~cm}^{-2} \mathrm{~s}^{-1}$ at collision optics. This corresponds to a $\beta_{\text {peak }}$ in the triplets of $9.1 \mathrm{~km}$, which is about the maximum value allowing a region of good field quality in the 5 -cm-bore triplet quadrupoles. It is necessary to increase the triplet bore in order to achieve $\beta^{*}=0.25 \mathrm{~m}$.

Figure 4 shows the $\beta$-functions and vertical dispersion $\eta_{y}$ across one IR at collision conditions with $\beta^{*}=0.5 \mathrm{~m}$. The horizontal dispersion is cancelled everywhere in the IR by use of dispersion suppressors at the ends of the adjacent arcs. The total phase advance across the IR is $\mu_{0}=3.375 \times 2 \pi$ in both planes. The distribution of the magnets is shown at the top of Figure 4.

The maximum beam size in the triplets at collision with $\beta^{*}=0.5 \mathrm{~m}$ is 5.5 times larger than anywhere in the arcs. The contribution to the linear chromaticity from 
both triplets is about -39 units compared with -124 units contributed by all quadrupoles in the arcs. In the rest of the IR, however, the $\beta$-functions are suppressed to a moderate level by optimizing the locations and strengths of the IR quadrupoles.

At injection energy the beam emittance is 10 times larger than at collision; thus, a much smaller $\beta_{\text {peak }}$ value is allowed under these conditions. The optimum value for $\beta^{*}$ at injection is chosen to be $7 \mathrm{~m}$, for which both the linear chromaticity of the IR and $\beta_{\text {peak }}$ are a minimum. The transfer matrix and the phase advance across an entire IR are the same for both collision and injection conditions. The lattice functions at injection are shown in Figure 5.

The quadrupole doublet QL4 and QL5 provides the final focusing at the secondary focus. These quadrupoles can be either $(F, D)$ or $(D, F)$ in a given plane. The difference is that one option gives larger beta peak within the tuning section at injection configuration, and the other at collision. We have chosen the second option in order to maximize the dynamic aperture at injection when the emittance is large.

Due to antisymmetry, the sum of $x$ and $y$ phase advances across each half IR is fixed and equal to

$$
\mu_{x l}+\mu_{y l}=\mu_{x r}+\mu_{y r}=\mu_{0},
$$

where

$$
\mu_{x l}=\mu_{y r}=\mu_{1}, \quad \mu_{y l}=\mu_{x r}=\mu_{2}, \quad \mu_{1}+\mu_{2}=\mu_{0},
$$

and the subscripts $l, r$ refer to the left and right half of the IR, respectively. However, the values $\mu_{1}$ and $\mu_{2}$ can be adjusted by appropriate choice of the gradients of quadrupoles in the tuning section. In our design we have chosen the following values for the phase advance across half an IR:

$$
\mu_{1}=1.875 \times 2 \pi, \quad \mu_{2}=1.5 \times 2 \pi .
$$

Taking into account Equation (9) and phase advance of $90^{\circ}$ per cell in the arcs, the above choice gives us a specific value of phase advance between the triplet and $\mathrm{F}$ or D quadrupoles in the regular cells in the arcs, viz.

$$
\mu_{x}^{F}-\mu_{x}^{t r p l}=n_{x} \frac{\pi}{2}, \quad \mu_{y}^{D}-\mu_{y}^{t r p l}=n_{y} \frac{\pi}{2},
$$

where $n_{x}$ and $n_{y}$ are integers. These particular phase advances have been chosen to optimize the local correction systems for the IR at collision optics. Two correction systems for the IR have been designed, namely, the local nonlinear chromaticity correction system [17] and the crossing angle system [14]. Both of them use a set of correctors placed next to $\mathrm{F}$ and $\mathrm{D}$ quadrupoles in the regular cells adjacent to the IRs. With the above choice given in Equation (13), these correctors are at optimal positions to compensate for the perturbations generated in the triplets.

There is a section of 7 regular half cells, named the hinge, that connects two IRs in the cluster. It contains horizontal dipoles that bend the orbit by $\approx 40.5 \mathrm{mrad}$. These 
dipoles are distributed so that the local perturbation of the dispersion generated by them stays within this section. The phase advance across the hinge is $0.875 \times 2 \pi$; therefore, the phase advance between the IPs in the two IRs in the cluster is $\pi / 2+8 \pi$. The phase advance between any two equivalent points in the first and second IRs has this value if the optics of both IRs are the same. This choice of phase ensures that the $\beta$-wave induced either by momentum offset or by systematic gradient errors in identical quadrupoles in the two IRs stays confined within the identical IRs. This is especially important for the low- $\beta$ IR configuration because it significantly reduces the strong nonlinear chromaticity generated by the final focus triplets.

With different values of $\beta^{*}$ at the first and second IPs (asymmetric configuration). the phase advance between the equivalent triplets in two IRs is still $\pi / 2+8 \pi$, but the $\beta_{\text {peak }}$ values are different. In this case, the chromatic and systematic gradient errors in the triplets are only partially compensated. To ensure compensation of the chromatic beta beat and associated nonlinear chromatic tune shift induced by the triplets in an asymmetric configuration, a local chromaticity correction system has been designed [17].

The whole cluster, which includes the two IRs, the hinge, the utility section, and the interconnect sections, has a unit transfer matrix. Hence, the idealized cluster does not amplify the effects of systematic magnetic errors in the arcs.

\section{Beta Squeeze}

As mentioned in Section 4, different optical configurations are required at injection and collision conditions. Injection optics is to be used at injection energy and while accelerating the beams. Shortly after the collision energy is reached, a smooth transition to collision optics, called $\beta$-squeeze, has to be made. Six independent gradients (currents) of the quadrupoles in the tuning section are simultaneously varied until the desired $\beta^{*}$ at collision is attained. The optical requirements for the $\beta$-squeeze have been indicated in Section 3.4. Figure 6 shows the variation of currents during the transition from $\beta^{*}=7 \mathrm{~m}$ to $\beta^{*}=0.25 \mathrm{~m}$ as a function of $\beta^{*}$ with $L^{*}=20.5 \mathrm{~m}$. The transfer matrix across the IR and the phase advances across each half of an IR are kept constant between injection and collision configurations. This guarantees that the rest of the machine is unaffected in the absence of errors.

Figure 6 shows that only the power supply across QL8 will have a large swing from injection to collision optics. The other quadrupoles may use weaker power supplies. It is important that there be no reversal of polarity of any tuning currents during the $\beta$-squeeze, which makes this procedure robust.

Figure 6 also shows a slow change of the currents in the high- $\beta^{*}$ part of the $\beta$-squeeze and a fast change in the low- $\beta^{*}$ part. The linear chromaticity of the IR behaves similarly during this transition. With linear dependence of $\beta^{*}$ on time, this would result in a much larger rate of change of current $d I / d t$ in the tuning quadrupoles and correction sextupoles at the end of the $\beta$-squeeze than at the beginning. There are limitations on the maximum values of $|d I / d t|$ in superconducting magnets. Therefore, 
a nonlinear time dependence of $\beta^{*}$ must be specified to make the variations of currents and the IR chromaticity more uniform with time.

A qualitative solution for the time dependence of $\beta^{*}$ can be found by a simple consideration of the IR chromaticity $\xi_{I R}$. At a low- $\beta^{*}$ configuration we can roughly neglect the contribution to $\xi_{I R}$ from the quadrupoles other than final focus triplets. Taking into account Equation (8) this leads to the following qualitative dependence:

$$
\xi_{I R}(t)=\frac{1}{4 \pi} \int_{I R} \beta K d s \approx \frac{1}{4 \pi} \int_{t r p l} \beta K d s \propto \beta_{\text {peak }}(t) \propto \frac{1}{\beta^{*}(t)},
$$

where $K=B^{\prime} /(B \rho)$, the gradient divided by the magnetic rigidity.

Assuming a constant rate of change of $\xi_{I R}$ with time, we find that $\beta^{*}$ has to be an inverse linear function of time:

$$
\beta^{*}(t)=\frac{1}{A_{l}+B_{l} t}
$$

where the coefficients $A_{l}$ and $B_{l}$ can be determined in terms of injection and collision values of $\beta^{*}$ and the duration $T$ of the $\beta$-squeeze. This function, indeed, works quite well at low values of $\beta^{*}$, but less so at high values of $\beta^{*}$, where the above assumptions (14) are not satisfied. A quantitative study showed that inverse quadratic and exponential functions lead to a satisfactory time dependence of the tuning currents and IR chromaticity in the full range of $\beta^{*}$ :

$$
\begin{gathered}
\beta^{*}(t)=\frac{1}{A_{q}+B_{q} t+C_{q} t^{2}}, \\
\beta^{*}(t)=A_{e} \exp \left(-t / B_{e}\right)+C_{e},
\end{gathered}
$$

where $A, B$, and $C$ are constant parameters.

Taking the exponential function (17) as a basis and minimizing both the rate of change of current in the tuning quadrupoles and the rate of change of chromaticity through the IR leads to the following variation:

$$
\beta^{*}(t)=6.8 \exp (-t / 20.36)+0.2
$$

The plot of tuning currents versus time with the above time dependence is shown in Figure 7. The duration of the squeeze is chosen to be $T=100$ seconds, which is almost the least time consistent with magnet specifications for maximum allowed value of $|d I / d t|$; it is also the duration used at FNAL. It can be easily increased if necessary. Figure 8 shows the rate of change of current through each tuning quadrupole during the squeeze. 


\section{Different $\mathrm{L}^{*}$}

The experimental requirements are expected generally to be different in the four IRs in the Collider, and they might change in the future. Therefore, the IR optics should be flexible enough to meet different requirements, such as space for the detector and beam size at the IP. In the present solution, $L^{*}$ can be set to any value in the range between $20.5 \mathrm{~m}$ and $90 \mathrm{~m}$ by a simple modification. The IR configuration has been designed to provide sufficient free space between the $M=-I$ section and the BV1 dipole. To increase the value of $L^{*}$, the triplet and adjacent pair of dipoles are moved together from the IP, by the required distance towards the $M=-I$ section while the other IR magnets stay in the same positions. The lengths of the triplet quadrupoles are adjusted to: 1) keep the secondary focus at the same distance from the IP, and 2) minimize $\beta_{\text {peak }}$ in the triplet. As $L^{*}$ increases, the triplets get shorter because of the longer focal length. This is shown in Figure 9.

For any given $L^{*}$, we choose the lowest $\beta^{*}$ at collision that corresponds to $\beta_{\text {peak }} \approx$ $9 \mathrm{~km}$ in the triplet. Consequently, $\beta^{*}$ increases with $L^{*}$. Its behavior is shown in Figure 10. Injection optics requires a minimum of both $\beta_{\text {peak }}$ and chromaticity of the IR.

It is clear that for a new $L^{*}$ the range of $\beta^{*}$ values is changed. However, it can be shown that under the above conditions, the range of $\beta$-functions at the secondary focus changes only slightly with $L^{*}$, as long as the distance from the IP to the SF is large compared to the distance from the IP to the effective center of the triplet. This is important, because the range and behavior of the tuning currents stay almost the same for any $L^{*}<90 \mathrm{~m}$. Practically this means that for a new value of $L^{*}$, the new range of $\beta^{*}$ values has to be substituted in Figure 6, but the curves of tuning currents have to be adjusted very little.

Table 1 shows the relevant parameters for the four values of $L^{*}$ chosen. The values of $34.55 \mathrm{~m}$ and $56.90 \mathrm{~m}$ were chosen because in these cases two of the quadrupoles in the triplet have the same length. The two smaller values of $L^{*}$ can be used for the low- $\beta$ IRs in the East cluster, and the other two values might be used for the IRs in the West cluster.

\begin{tabular}{|c||c|c||c|c|c|}
\hline \hline \multicolumn{1}{|c||}{$L^{*}(\mathrm{~m})$} & \multicolumn{3}{c||}{$\beta^{*}(\mathrm{~m})$} & \multicolumn{3}{c|}{ Triplet Quad Lengths $(\mathrm{m})$} \\
& Inj. & Coll. & QL1 & QL2 & QL3 \\
\hline 20.50 & 7.0 & 0.5 & 15.5650 & 11.8545 & 13.1715 \\
\hline 34.55 & 11.0 & 0.7 & 12.6064 & 11.1793 & 12.6064 \\
\hline 56.90 & 23.0 & 1.1 & 10.2948 & 10.2948 & 11.8835 \\
\hline 90.00 & 40.0 & 1.95 & 8.6890 & 9.4405 & 11.2051 \\
\hline \hline
\end{tabular}

Table 1: Range of $\beta^{*}$ and triplet quadrupole lengths for four values of $L^{*}$. 
The $\beta$-functions at collision and tuning currents for the case of the largest $L^{*}=$ $90 \mathrm{~m}$ are shown in Figures 11 and 12, respectively.

\section{Magnet Parameters}

In the present design we use optimized, realistic parameters of the IR magnets. All the quadrupoles have $5-\mathrm{cm}$ bore and maximum gradient of $191 \mathrm{~T} / \mathrm{m}$. The 5 -cm-bore vertical dipoles are identical in design to the horizontal dipoles in the Collider. Only the dipoles BV1c located next to the triplets need the larger aperture of $8.7 \mathrm{~cm}$ to provide enough space for the separated beams.

The lengths and positions of the quadrupoles in the tuning section and in the $M=-I$ section were optimized to reduce the total length of magnets and number of different quadrupole lengths. Excluding the triplets, there are only 3 different lengths of IR quadrupoles: $8.0 \mathrm{~m}, 8.6 \mathrm{~m}$, and $10.2 \mathrm{~m}$.

The list of the magnet parameters for the IR configuration with $L^{*}=20.5 \mathrm{~m}$ is given in Table 2, where the range of the gradient variation during the $\beta$ squeeze is listed for the tuning quadrupoles. This covers a range in $\beta^{*}$ of $7.0 \mathrm{~m}$ at injection down to a value of $\beta^{*}=0.25 \mathrm{~m}$ at collision. The IR optics with other values of $L^{*}$ requires different lengths for the triplet quadrupoles, according to Figure 9.

\begin{tabular}{|c||c|c|c|}
\hline \hline Name & Length $(\mathrm{m})$ & $\begin{array}{c}\text { Gradient }(\mathrm{T} / \mathrm{m}) \\
\text { Field }(\mathrm{T}) \text { at } 20 \mathrm{TeV} / \mathrm{c}\end{array}$ & Bore (cm) \\
\hline \hline QL1 & 15.5650 & 190.885 & 5.0 \\
\hline QL2 & 11.8545 & 190.885 & 5.0 \\
\hline QL3 & 13.1715 & 190.885 & 5.0 \\
\hline QL4 & 10.2 & $155.2-174.9$ & 5.0 \\
\hline QL5 & 8.0 & $143.2-175.5$ & 5.0 \\
\hline QL6 & 8.0 & $128.2-160.5$ & 5.0 \\
\hline QL7 & 8.6 & $159.0-181.1$ & 5.0 \\
\hline QL8 & 8.0 & $23.4-169.2$ & 5.0 \\
\hline QL9 & 10.2 & $103.4-176.1$ & 5.0 \\
\hline QV & 8.0 & 190.885 & 5.0 \\
\hline BV1c & 15.8387 & 6.3997 & 8.7 \\
\hline BV1 & 14.9280 & 6.7901 & 5.0 \\
\hline BV2 & 12.4400 & 6.7901 & 5.0 \\
\hline \hline
\end{tabular}

Table 2: Magnet parameters for the low- $\beta$ IR: $L^{*}=20.5 \mathrm{~m}$. 


\section{Sensitivity to Triplet Errors}

The beam is specially sensitive to any errors in the triplet quadrupoles at collision conditions because of the high value of $\beta_{\text {peak }}$ and the large integrated gradient of these quadrupoles. Compared to the regular arc quadrupoles, the $\beta$-function in the triplets is up to 30 times larger, and each triplet quadrupole is more than twice as strong.

The errors can be in the quadrupole fields or in their alignments. Errors in the gradient give rise to a $\beta$-wave and a dispersion wave, and also change the tune of the machine. Multipole errors can change the widths of the nonlinear resonances and affect the long-term stability. The nature of the alignment error determines what parameters are affected. We list them below: $\Delta \beta, \Delta D$, and $\Delta \nu$ represent the change in $\beta$, the dispersion function, and the tune, respectively.

- Transverse Misalignment $\Rightarrow$ Closed Orbit Distortion, $\Delta D$.

- Longitudinal Misalignment $\Rightarrow \Delta \beta, \Delta D, \Delta \nu$.

- Rotational Misalignment about a transverse axis $\Rightarrow$ Closed Orbit Distortion, $\Delta D$.

- Rotational Misalignment about the longitudinal axis $\Rightarrow$ Change in the Coupling.

The effect of triplet errors is more complex compared to those in other quadrupoles, because the triplets are common for the two beams. Therefore, the errors affect both beams at the same time. First of all, the effect on each beam is different because of opposite focusing strengths seen by the two beams in a particular triplet quadrupole. Secondly, the crossing angle generates large orbit displacements in the triplets, which are different for the beams, thus resulting in larger multipole errors.

Rather than present a comprehensive study of the above effects in this section, we merely want to emphasize the importance of the control of the triplet errors and the need for their corrections. These problems are common for colliding machines with low- $\beta$ IRs because of the similarity of final focus regions in any design, and the effects are less pronounced for errors in the rest of the IR. In the following subsections we give some examples of the effect of triplet errors at collision compared to the effect due to regular arc quadrupoles.

\subsection{Gradient Errors}

For simplicity we will approximate the gradient errors by kicks due to thin quadrupoles and consider effects only to first order in the gradient error. Let $\Delta q$ represent the change in inverse focal length of a quadrupole with length $L$ due to a gradient error $\Delta B^{\prime}$, i.e.,

$$
\Delta q=\frac{\Delta B^{\prime} L}{(B \rho)} .
$$


$(B \rho)$ is the magnetic rigidity factor, which at SSC collision conditions is $66712.8 \mathrm{~T}-\mathrm{m}$. The change in the tune is

$$
\Delta \nu=\frac{\beta_{0} \Delta q}{4 \pi},
$$

while the maximum changes in the lattice functions are (see, e.g., [18])

$$
\begin{aligned}
\left(\frac{\Delta \beta}{\beta}\right)_{\max } & =\frac{\beta_{0} \Delta q}{2 \sin 2 \pi \nu} \\
(\Delta D)_{\max } & =\frac{\sqrt{\beta_{0} \beta(s)} \Delta q}{2 \sin \pi \nu} D_{0} .
\end{aligned}
$$

Here $\beta_{0}, D_{0}$ are the $\beta$-function and horizontal dispersion in the quadrupole with the gradient error, while $\beta(s)$ is the $\beta$-function at the location where the maximum change occurs. In Table 3 we compare the effects of gradient error in a triplet quadrupole of gradient $191 \mathrm{~T} / \mathrm{m}$, length $11.85 \mathrm{~m}$, and $\beta_{0}=9 \mathrm{~km}$ with that in a quadrupole of gradient $221 \mathrm{~T} / \mathrm{m}$, length $=4.86 \mathrm{~m}$, and $\beta_{0}=300 \mathrm{~m}$, which is placed in a regular cell in the arcs. We assume a relative gradient error of $10^{-4}$ and a fractional tune equal to 0.4 .

\begin{tabular}{|c||c|c|}
\hline & Triplet Quadrupole & Regular Quadrupole \\
\hline \hline$\Delta \nu$ & $2.4 \times 10^{-3}$ & $4 \times 10^{-5}$ \\
\hline$(\Delta \beta / \beta)_{\max }$ & 0.026 & 0.0004 \\
\hline \hline
\end{tabular}

Table 3: Comparison of the effects of a quadrupole gradient error $\Delta B^{\prime} / B^{\prime}=10^{-4}$ in triplet and regular quadrupoles.

For the particular quadrupole parameters and $\beta$-functions, the effect due to the triplet quadrupole is about 63 times larger. The nominal horizontal dispersion $D_{0}$ in the triplets is suppressed; therefore, ideally there is no change of the dispersion due to triplet gradient errors. It could be generated mostly due to errors in the arcs.

\subsection{Misalignment Errors}

The following are examples of misalignment errors:

- Transverse Misalignment (TM). The maximum closed orbit shift $t_{\max }$ due to an alignment error of $\Delta t$ in a quadrupole of length $L$ is

$$
t_{\max }(s)=\frac{\sqrt{\beta(s) \beta_{0}}}{2 \sin \pi \nu_{t}} \frac{B^{\prime} L}{(B \rho)} \Delta t,
$$

where $t$ denotes either of the transverse directions $x$ or $y, \beta_{0}$ is the $\beta$-function in the misaligned quadrupole, and $\beta(s)$ is the $\beta$-function at the location of the maximum shift. Clearly, a misaligned triplet quadrupole produces about one order of magnitude greater orbit deviations than an arc quadrupole. 
- Longitudinal Misalignment (LM). An error of this kind distorts the $\beta$-function and changes the tune. These changes depend on the $\beta$-functions at the beginning and the end of the quadrupole, $\beta_{b}$ and $\beta_{e}$, respectively. For a longitudinal misalignment of $\Delta s$, these are given by

$$
\begin{aligned}
\left(\frac{\Delta \beta}{\beta}\right)_{\max } & \approx \frac{1}{2 \sin 2 \pi \nu} \sqrt{\left(\beta_{e}-\beta_{b}\right)^{2}+4 L^{2}} \frac{B^{\prime}}{(B \rho)} \Delta s \\
\Delta \nu & =\frac{\left(\beta_{e}-\beta_{b}\right)}{4 \pi} \frac{B^{\prime}}{(B \rho)} \Delta s .
\end{aligned}
$$

There is also a dispersion wave generated by such a misalignment of a triplet quadrupole, but it will be a small effect for the reasons mentioned earlier. The above expressions show that the main effect is generated by the difference in values of $\beta$-functions at the two ends of the quadrupole. It is a small effect in the regular arc quadrupoles where $\beta$ is nearly symmetric around the quadrupole center. In the triplets the $\beta$-function may change significantly from end to end, so better precision is required for their longitudinal alignment.

- Rotational Misalignment about a transverse axis (RTM). If a quadrupole is rotated, for instance, in the $(x, s)$ plane, then a dipole field is created along the $y$ axis leading to a closed orbit shift along the $x$ axis. For an angular misalignment by $\Delta \theta_{t}$ in the plane $(t, s)$, the maximum closed orbit shift is

$$
t_{\max }(s) \approx \frac{\sqrt{\beta(s)}}{2 \sin \pi \nu_{t}} \frac{B^{\prime}}{(B \rho)} I \Delta \theta_{t} .
$$

$I$ is the following integral,

$$
I=\int_{-L / 2}^{L / 2} s \sqrt{\beta_{0}(s)} d s
$$

where $\beta_{0}$ is the $\beta$-function within the quadrupole. Here we neglect the change of phase advance across the quadrupole, which is particularly very small in the triplets. The maximum of the orbit shift will occur when a focusing quadrupole is misaligned, in which case $\beta_{0}$ is

$$
\begin{aligned}
\beta_{0}(s) & =a \cos 2 \sqrt{K} s+b \sin 2 \sqrt{K} s+c \\
a & =\frac{1}{4 \sqrt{K} \sin \sqrt{K} L}\left[\beta_{b}^{\prime}-\beta_{e}^{\prime}\right] \\
b & =\frac{1}{2 \sin \sqrt{K} L}\left(\beta_{e}-\beta_{b}\right) \\
c & =\beta_{b}-(a \cos \sqrt{K} L-b \sin \sqrt{K} L),
\end{aligned}
$$

where $K=B^{\prime} /(B \rho)$ and $\beta_{b}^{\prime}, \beta_{e}^{\prime}$ are the slopes of the $\beta$-function at the beginning and end of the quadrupole, respectively. The important coefficient is $b$, which 
depends on the difference of the $\beta$-functions at the two ends. If $b=0, \sqrt{\beta_{0}(s)}$ is an even function of $s$ and the integral $I$ vanishes. In a regular quadrupole $\beta_{b} \approx \beta_{e}$ and, consequently, such a rotational misalignment produces a small effect on the closed orbit. Within the triplet the greatest contribution will come from the quadrupole within which the $\beta$-function changes the most. The integral $I$ can be evaluated numerically.

- Rotational Misalignment about the longitudinal axis (RLM). The effect of such an error is to couple the $x$ and $y$ motions. The strength of the coupling can be measured by the minimum tune difference $\Delta \nu_{\text {dif }}$ between the two eigentunes. If the quadrupole is rotated by an angle $\Delta \phi$ about the $s$ axis, this difference is given by [18]:

$$
\Delta \nu_{d i f}=\frac{\sqrt{\beta_{x} \beta_{y}}}{\pi} \frac{B^{\prime} L}{(B \rho)} \Delta \phi .
$$

$\beta_{x}, \beta_{y}$ can be taken as the average $\beta$-functions in the two planes within the misaligned quadrupole. Clearly a misaligned triplet quadrupole causes more than one order of magnitude greater effect than an arc quadrupole.

Table 4 compares the effects of the above errors in a triplet quadrupole where the $\beta$-function reaches its peak value with that of an alignment error in a standard quadrupole in an arc cell. The fractional part of the tune is taken to be 0.4 .

\begin{tabular}{|c|c||c|c|}
\hline Misalignment Error & Effect & Triplet Quadrupole & Regular Quadrupole \\
\hline \hline TM $(\Delta t=1 \mathrm{~mm})$ & Orbit Shift & $29.3 \mathrm{~mm}$ & $2.5 \mathrm{~mm}$ \\
\hline \multirow{2}{*}{ LM $(\Delta s=1 \mathrm{~mm})$} & $(\Delta \beta / \beta)_{\max }$ & $9.6 \times 10^{-3}$ & $3 \times 10^{-5}$ \\
\cline { 2 - 4 } & $\Delta \nu$ & $9 \times 10^{-4}$ & $4 \times 10^{-8}$ \\
\hline RTM $\left(\Delta \theta_{t}=1 \mathrm{mrad}\right)$ & Orbit Shift & $7.3 \mathrm{~mm}$ & $5 \times 10^{-3} \mathrm{~mm}$ \\
\hline RLM $(\Delta \phi=1 \mathrm{mrad})$ & $\Delta \nu_{\text {dif }}$ & $6.3 \times 10^{-2}$ & $7 \times 10^{-4}$ \\
\hline \hline
\end{tabular}

Table 4: Comparison of the effects of quadrupole misalignment errors.

The orbit changes have been evaluated in the arcs. Within the triplets the shift will be up to 5.5 times larger than that in the arcs. The effect increases, if all the triplet quadrupoles in four IRs are taken into account. It is clear, therefore, that possible beam losses most likely will happen in the triplet area, where the necessary protection is required.

The above results illustrate that especially the transverse and rotational misalignments of the triplet quadrupoles have to be controlled to a very high degree in order to avoid large perturbations on the beam. It is obvious that beam sensitivity to triplet errors increases at lower values of $\beta^{*}$. 


\section{Acknowledgements}

We acknowledge the important contributions to the early design of the Interaction Regions by D. Johnson, K. Steffen, and S. Peggs. We also thank K. Brown, A. Chao, D. Edwards, H. Edwards, M. Harrison, R. Talman, and T. Toohig for useful discussions. 


\section{References}

[1] F.M. Izrailev, S.I. Mishnev, and G.M. Tumaikin, "Numerical Experiments for a Determination of the Stochasticity Criterion in Beam-Beam Interactions," Preprint INP 77-43, Novosibirsk, 1977.

[2] P.E. Faugeras et al., "The Low-Beta Insertions of the SPS Proton-Antiproton Collider," Proceedings of the 12th International Conference on High-Energy Accelerators, pp. 232-234, Batavia, Illinois, August 11-16, 1983.

[3] A.D. McInturff et al., "The Fermilab Collider D0 Low $\beta$ System," Proceedings of the European Particle Accelerator Conference, pp. 1264-1266, Rome, June 7-11. 1988.

[4] W. Scandale, "The Lattice of the LHC: Version 1," CERN Divisional Report SL/91-03 (AP), LHC Note No. 139, Geneva, January 1991.

[5] Report of the Reference Designs Study Group on the Superconducting Super Collider, U.S. Department of Energy, May 8, 1984.

[6] M.J. Syphers, "Horizontally Separated 1-in-1 Crossing Insertions," Report of the Workshop on Realistic SSC Lattices, pp. 15-26, SSC-SR-1015, Berkeley, October 1985.

[7] A. Garren, "A Design for Vertical Crossing Insertions," Report of the Workshop on Realistic SSC Lattices, pp. 31-34, SSC-SR-1015, Berkeley, October 1985.

[8] A. Garren, "Lattice of the SSC," Proceedings of The 13th International Conference on High-Energy Accelerators, pp. 74-77, Novosibirsk, June 1986.

[9] A. Garren and D. Johnson, "The 90 Degree (September 1987) SSC Lattice," SSC Report SSC-146, 1987.

[10] A. Garren and D. Johnson, "Status of the SSC Lattice Design," Proceedings of The European Particle Accelerator Conference, pp. 723-725, Rome, June 7-11, 1988.

[11] J.R. Sanford, D.M. Matthews, eds., Site-Specific Conceptual Design, SSCL-SR$1056,1990$.

[12] Y. Nosochkov et al., "Current Design of the SSC Interaction Regions," SSCLPreprint-368, May 1993; presented at 1993 IEEE Particle Accelerator Conference, Washington, May 17-20, 1993.

[13] Y. Nosochkov, "Design of the SSC Medium-Beta Interaction Regions," SSCLPreprint-528, June 1993; presented at Workshop on B Physics at Hadron Accelerators, Snowmass, Colorado, June 21-July 2, 1993. 
[14] Y. Nosochkov and D. Ritson, "The Provision of IP Crossing Angles for the SSC," SSCL-Preprint-367, May 1993; presented at 1993 IEEE Particle Accelerator Conference, Washington, May 17-20, 1993.

[15] R. Stiening, F. Pilat, private communication.

[16] D. Ritson, private communication.

[17] T. Sen et al., "Chromaticity Correction for the SSC Collider Rings," SSCLPreprint-413, May 1993; presented at 1993 IEEE Particle Accelerator Conference, Washington, May 17-20, 1993.

[18] D. Edwards and M.J. Syphers, An Introduction to the Physics of High Energy Accelerators, John Wiley \& Sons, NY, 1993. 


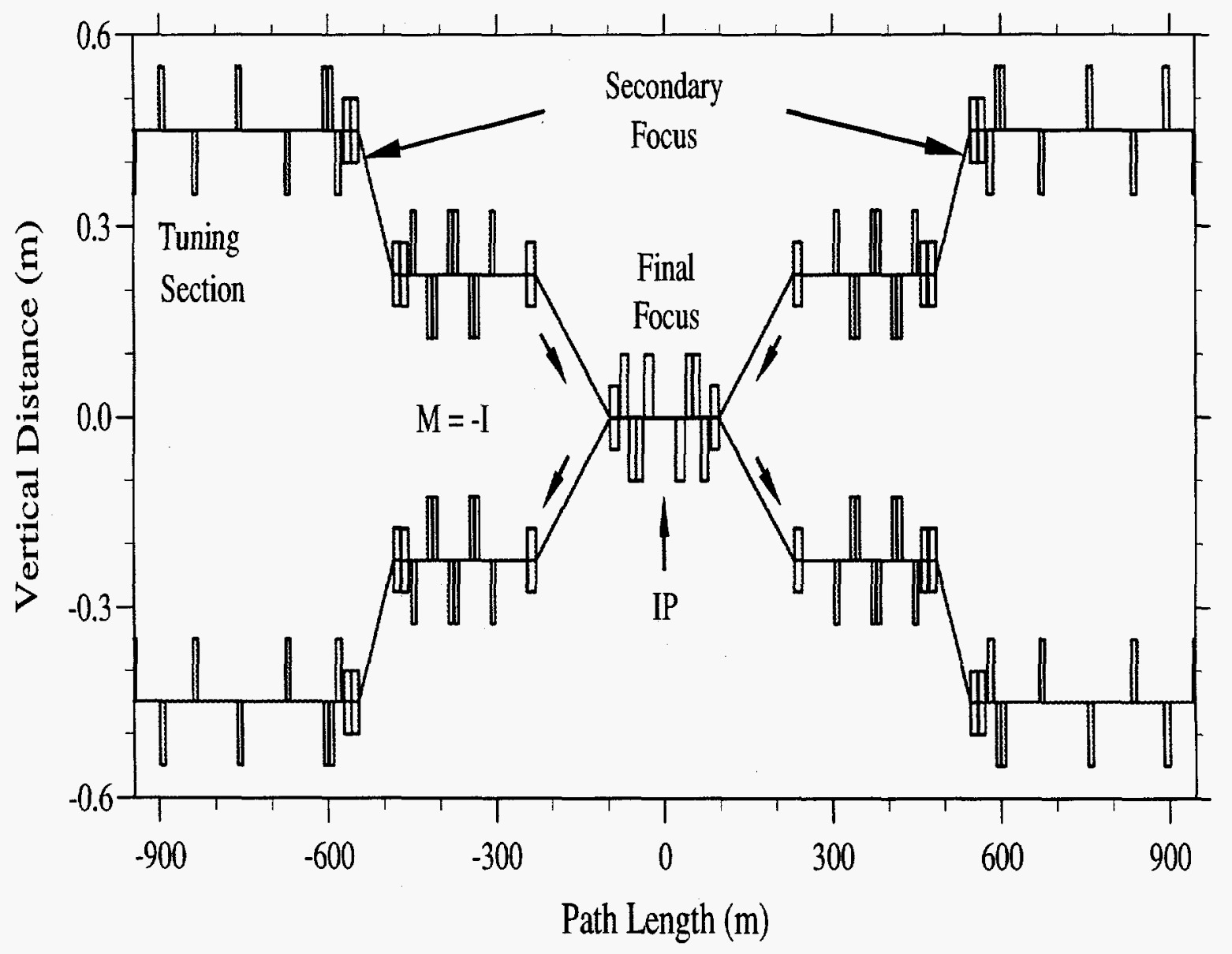

Figure 1: Vertical view of an IR. 


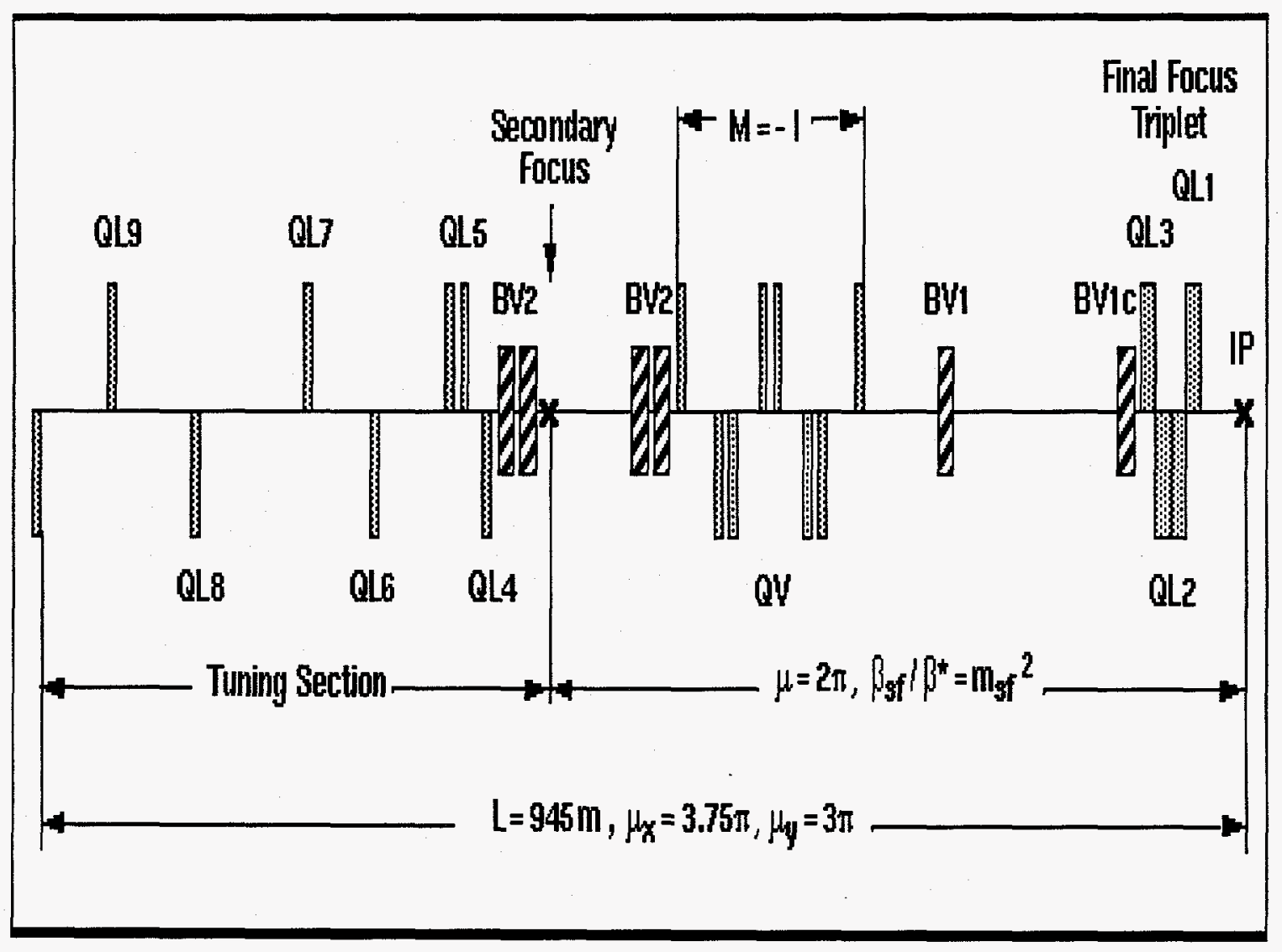

Figure 2: Optical modules in half of an IR. 


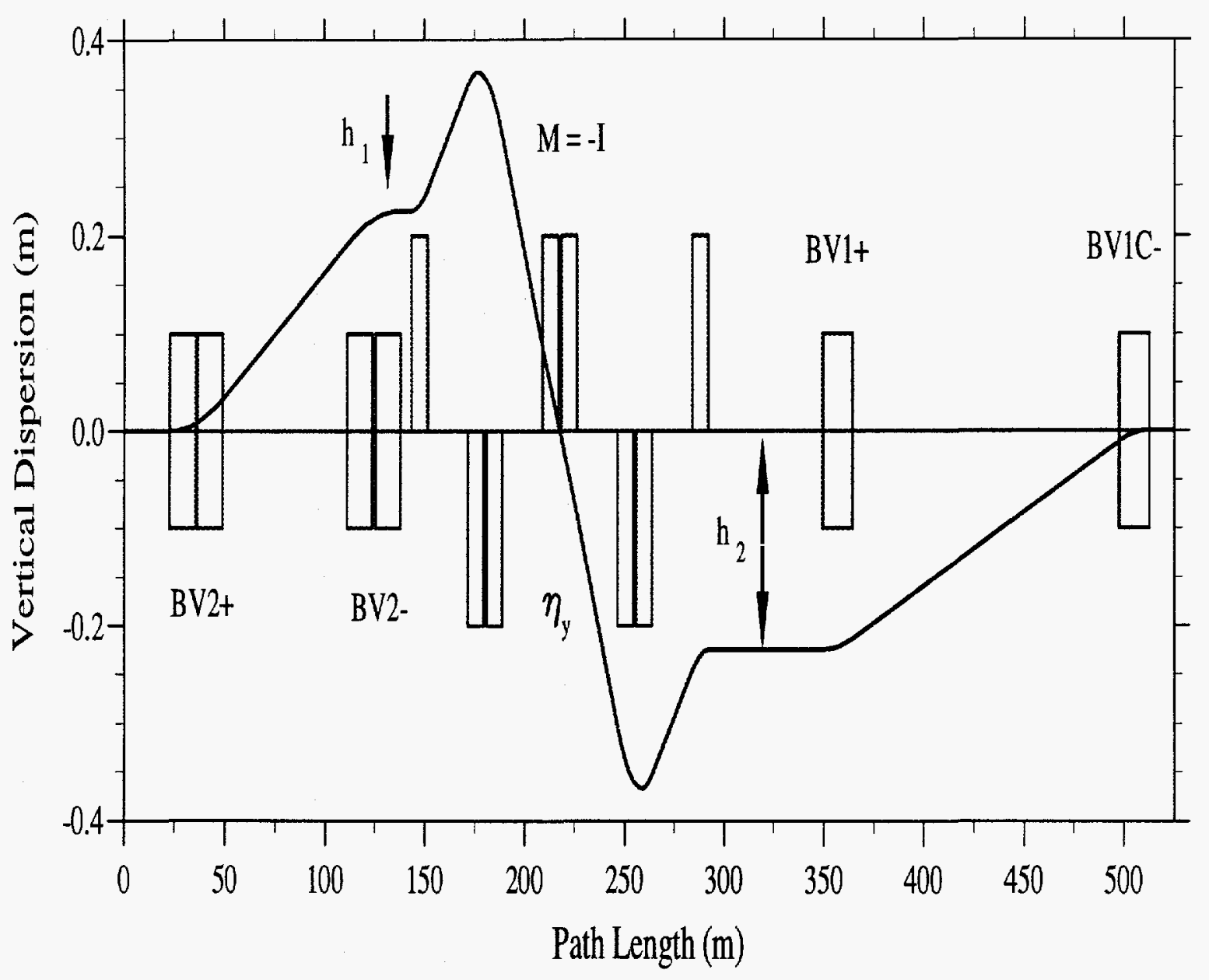

Figure 3: Cancellation of vertical dispersion with $M=-I$ section. 


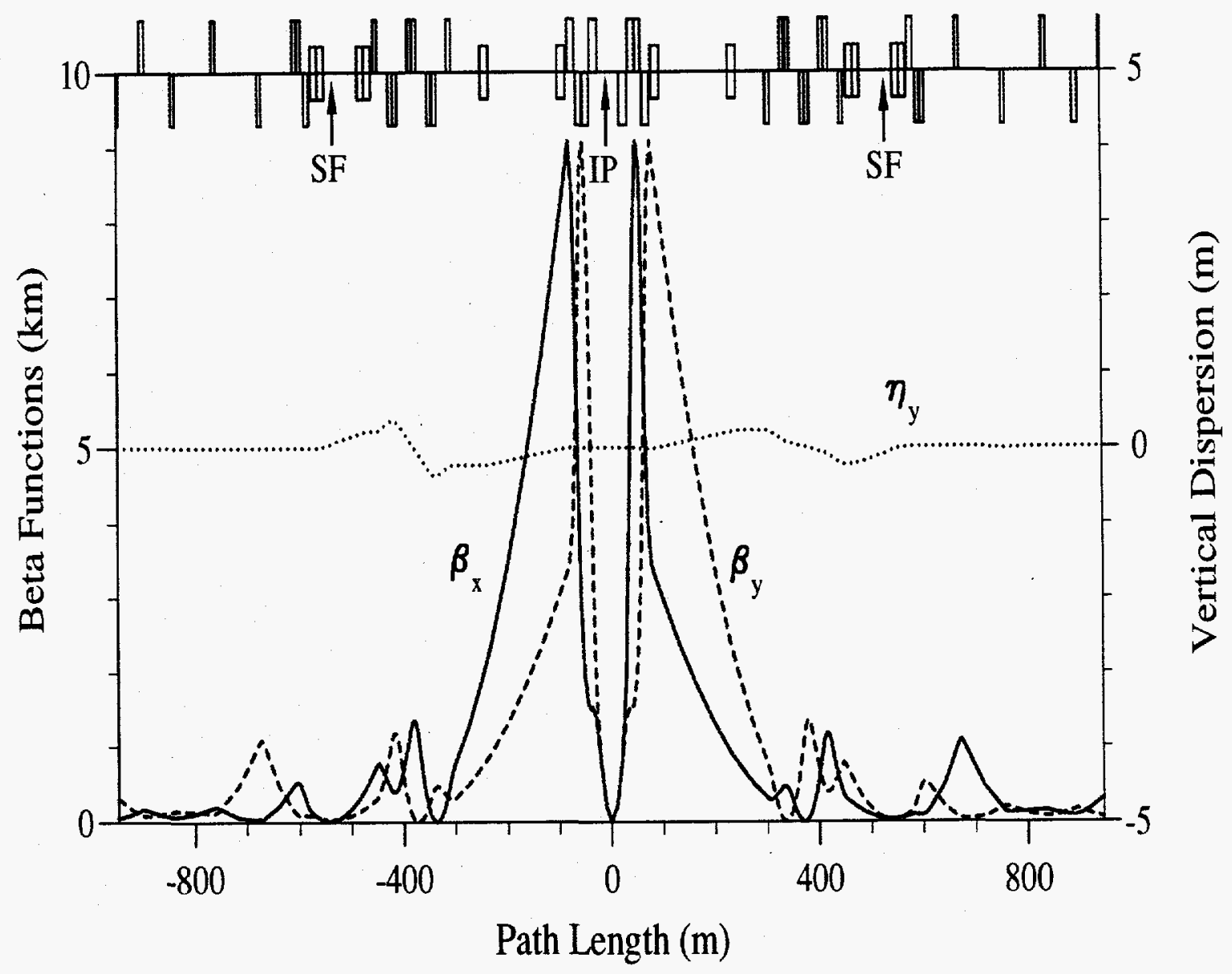

Figure 4: Lattice functions in the low- $\beta$ IR at collision: $\beta^{*}=0.5 \mathrm{~m}, L^{*}=20.5 \mathrm{~m}$. 


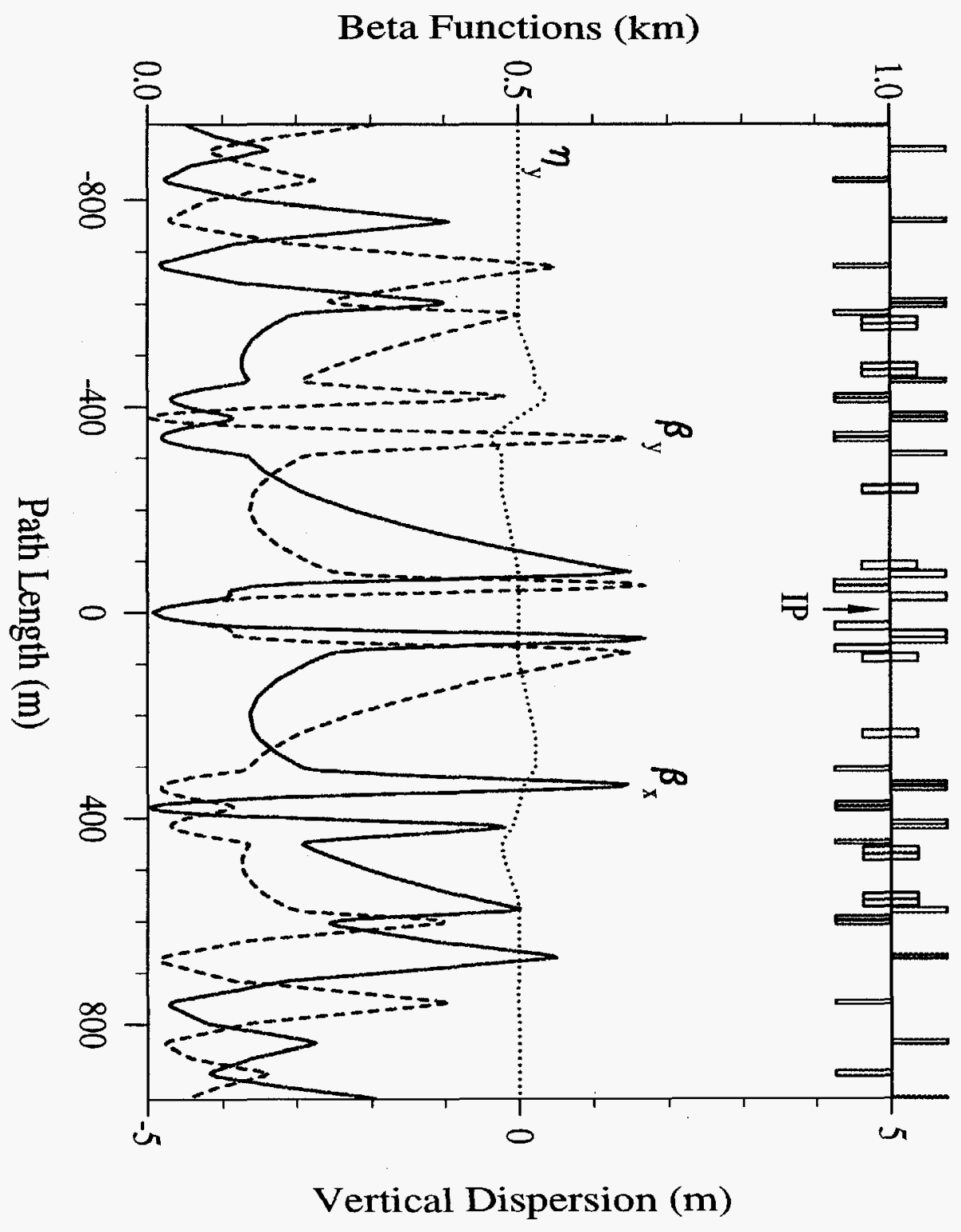




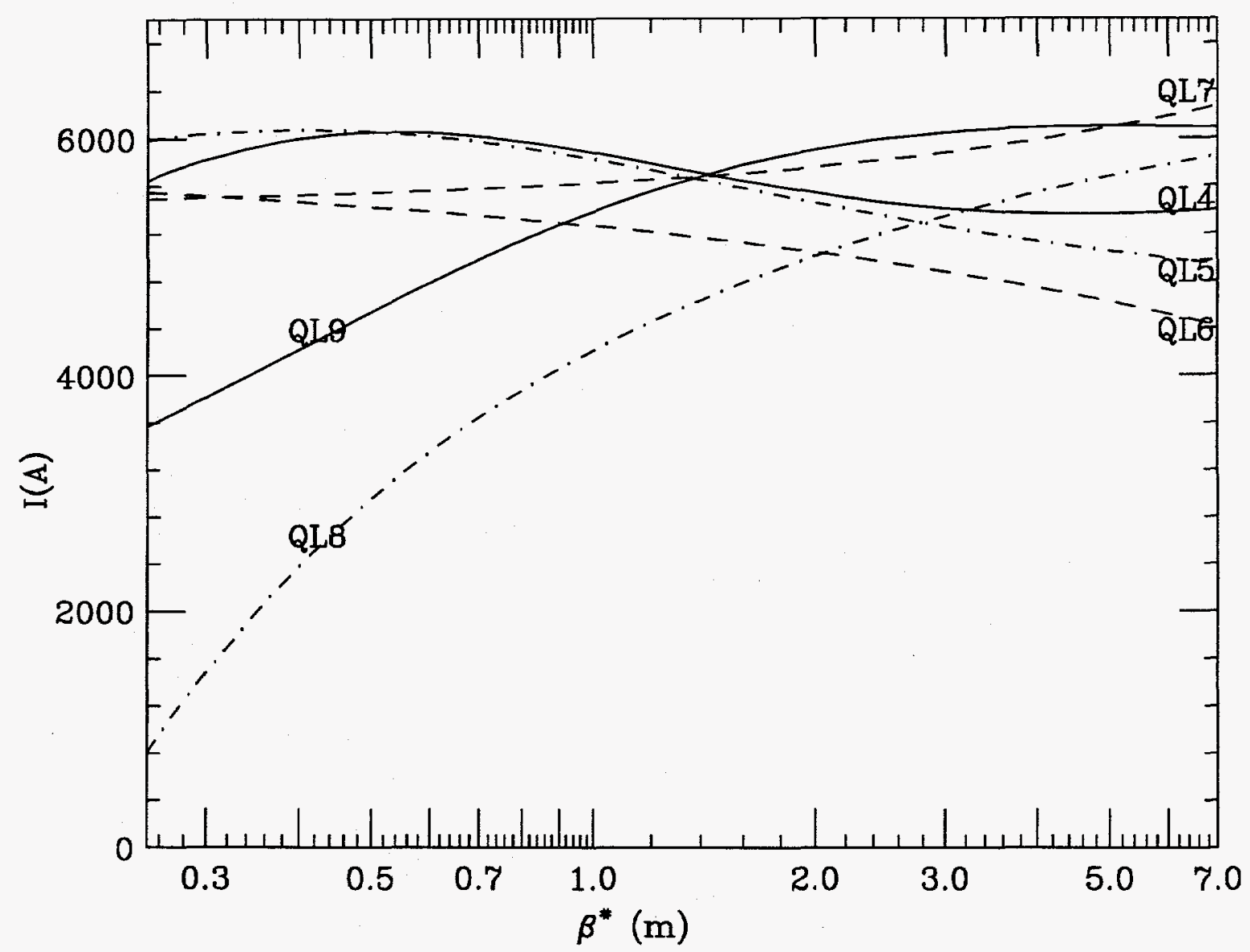

Figure 6: Tuning currents vs $\beta^{*}$ for a low- $\beta$ IR optics: $L^{*}=20.5 \mathrm{~m}$. 


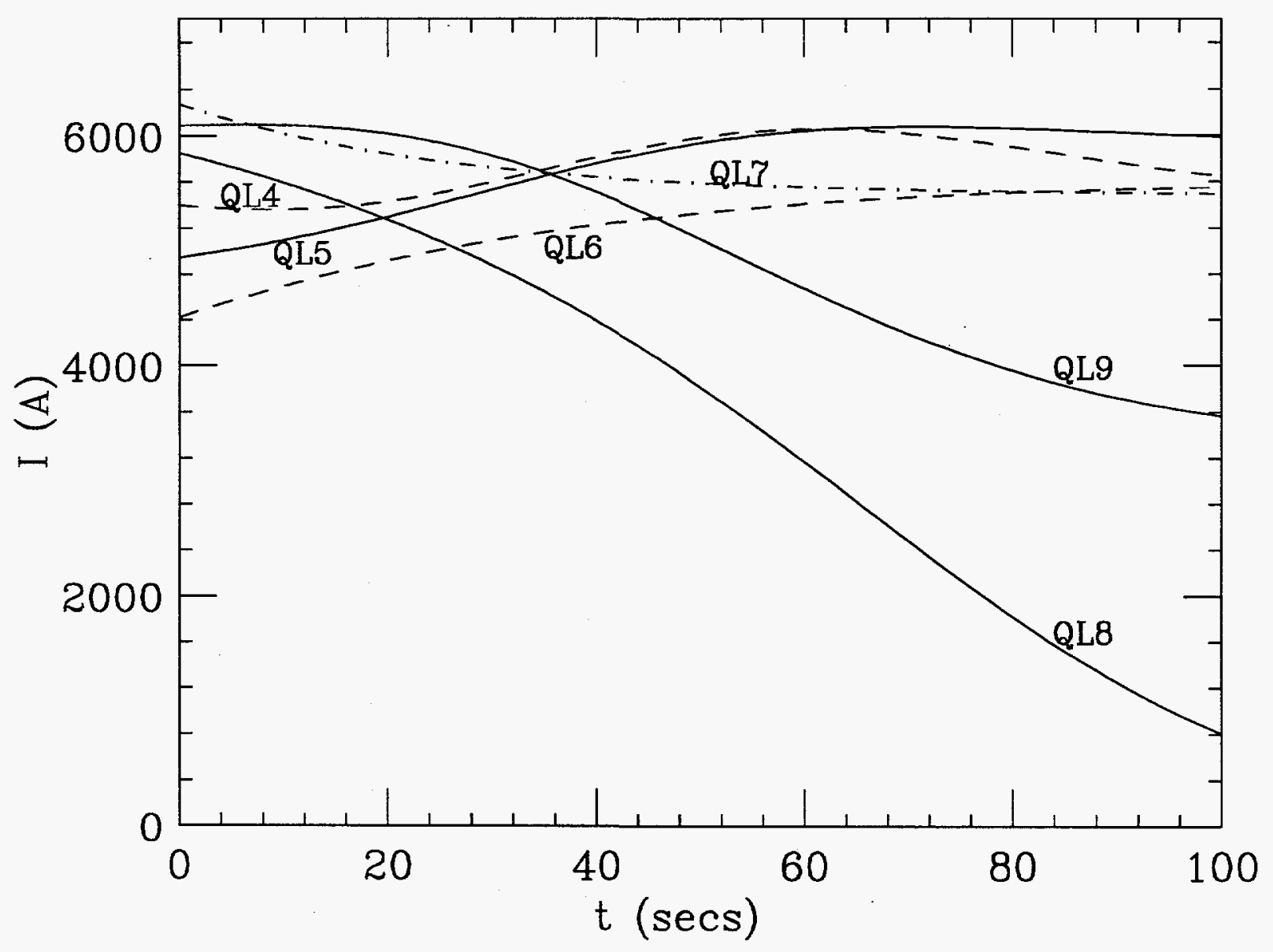

Figure 7: Tuning currents vs time for a low- $\beta$ IR optics: $L^{*}=20.5 \mathrm{~m}$. 


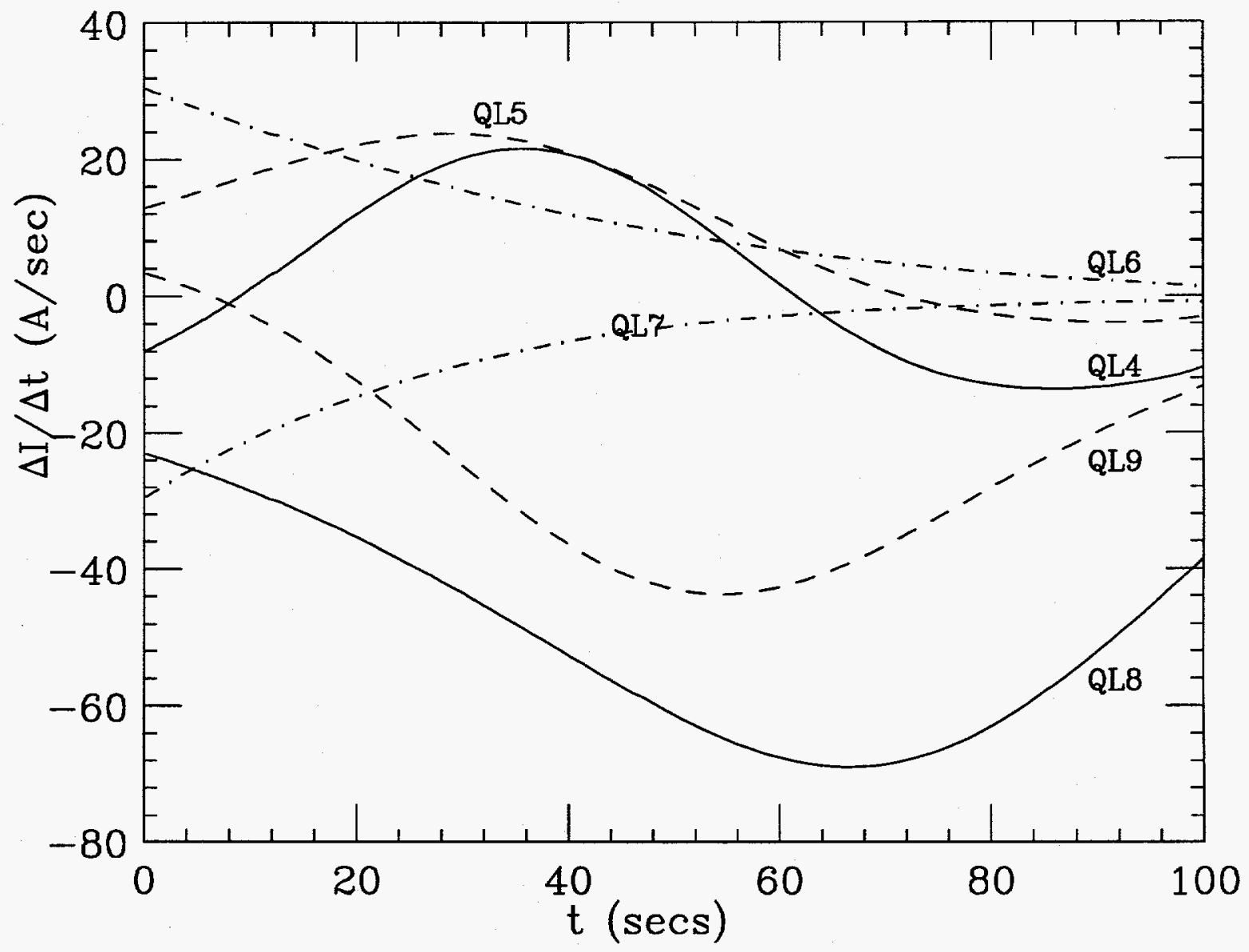

Figure 8: Rate of change of current during $\beta$-squeeze: $L^{*}=20.5 \mathrm{~m}$. 


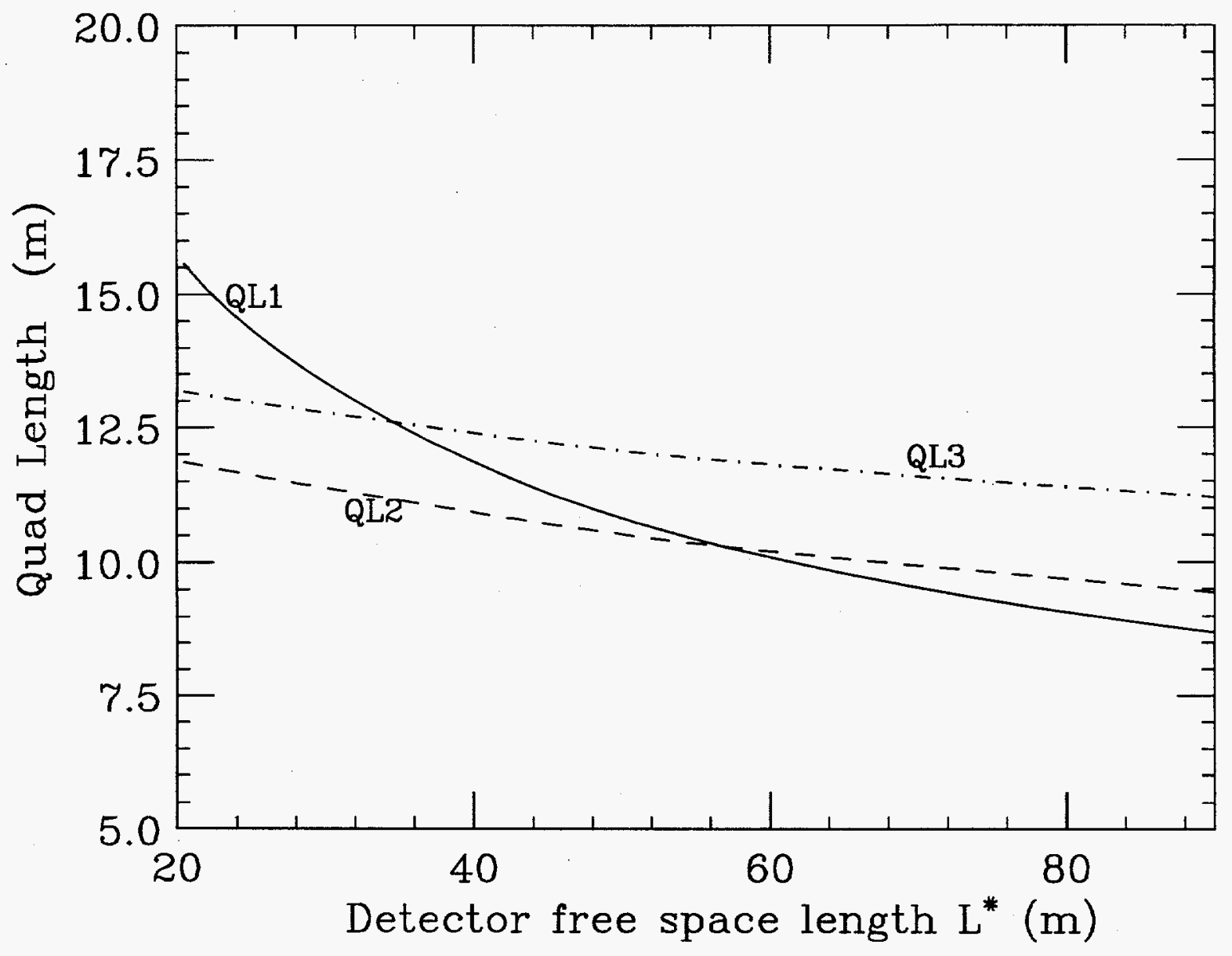

Figure 9: Triplet quadrupole lengths vs $L^{*}$. 


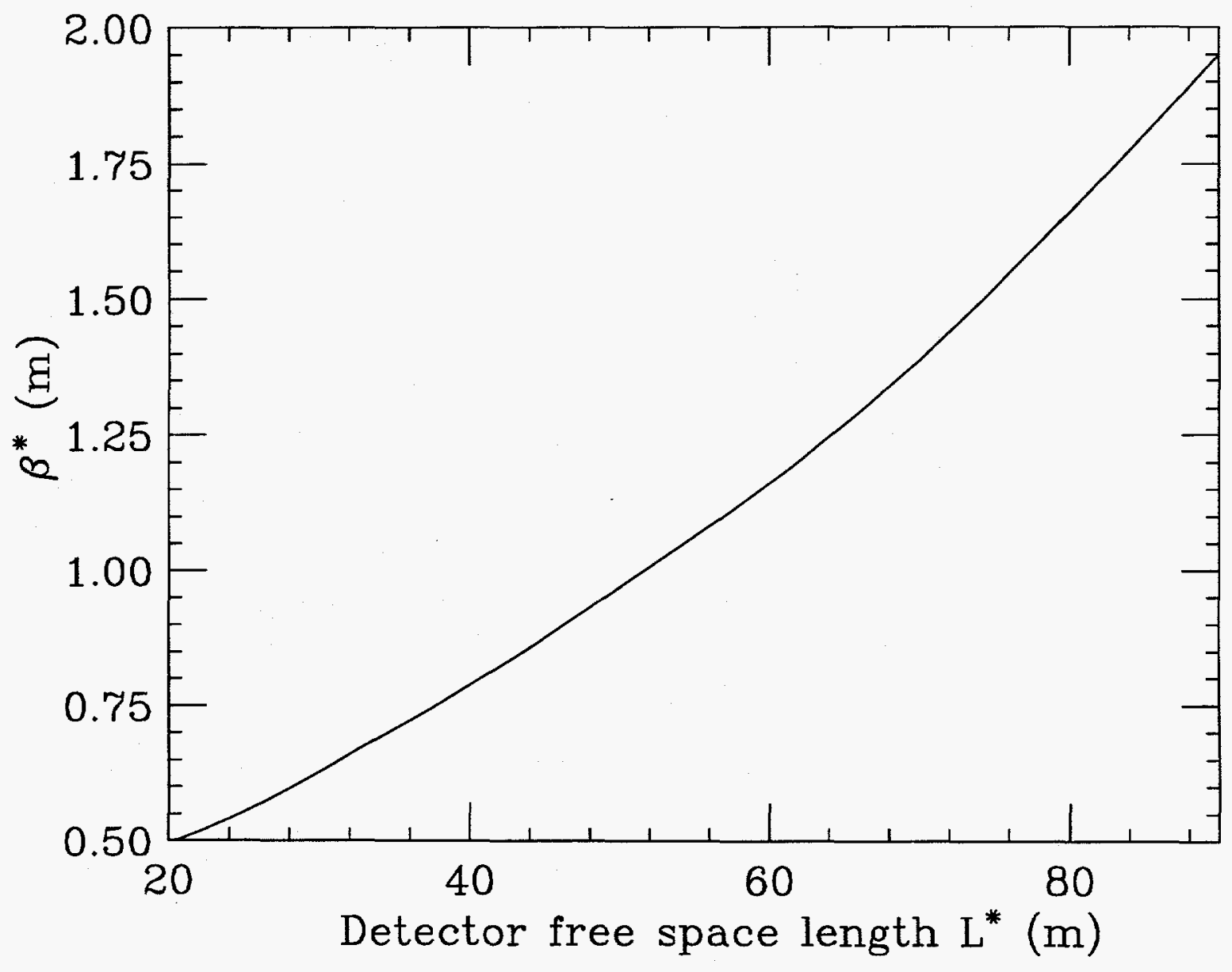

Figure 10: The lowest $\beta^{*}$ vs $L^{*}: \beta_{\text {peak }} \approx 9 \mathrm{~km}$. 


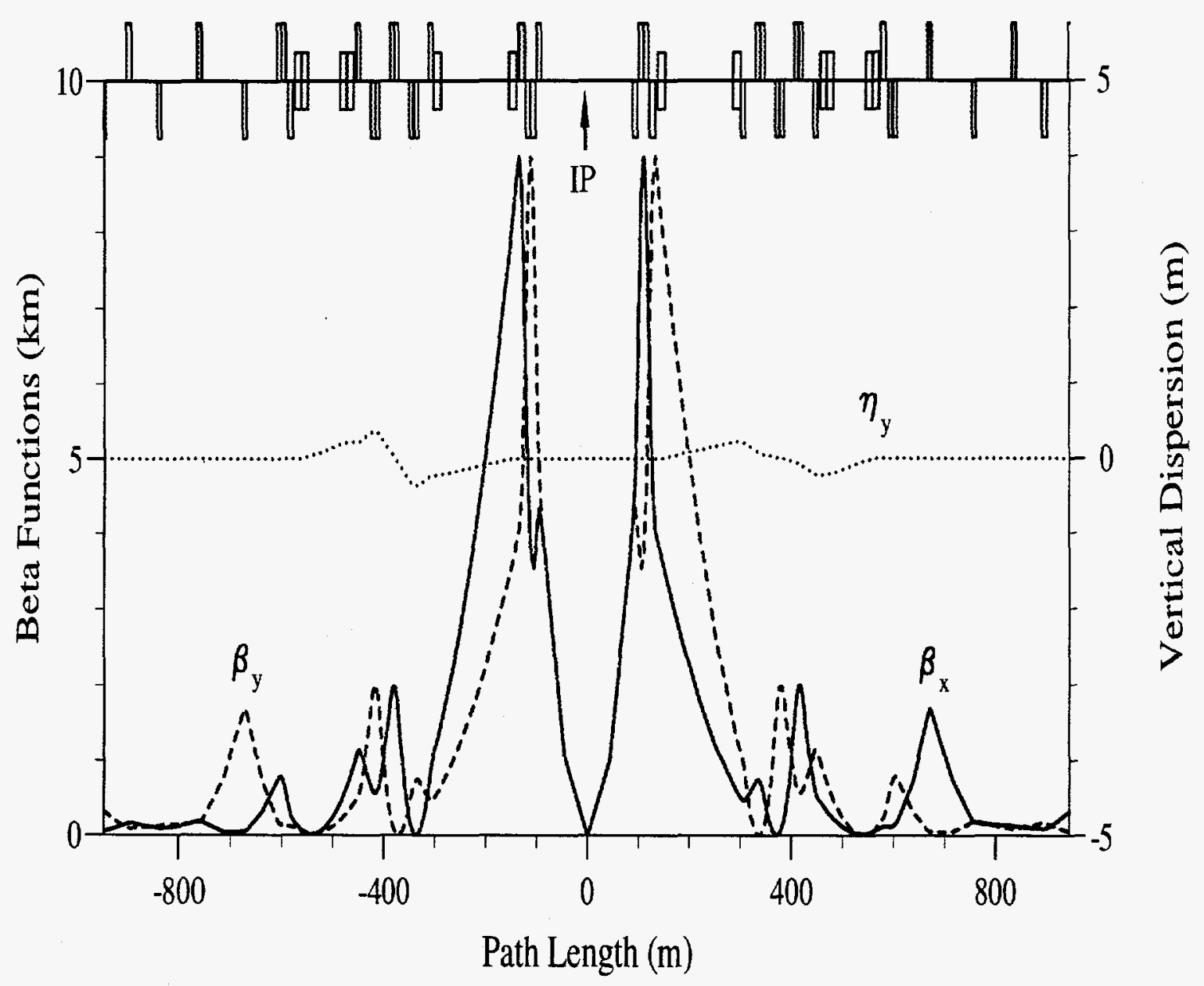

Figure 11: Lattice functions in the medium- $\beta$ IR at collision: $\beta^{*}=1.95 \mathrm{~m}, L^{*}=90 \mathrm{~m}$. 


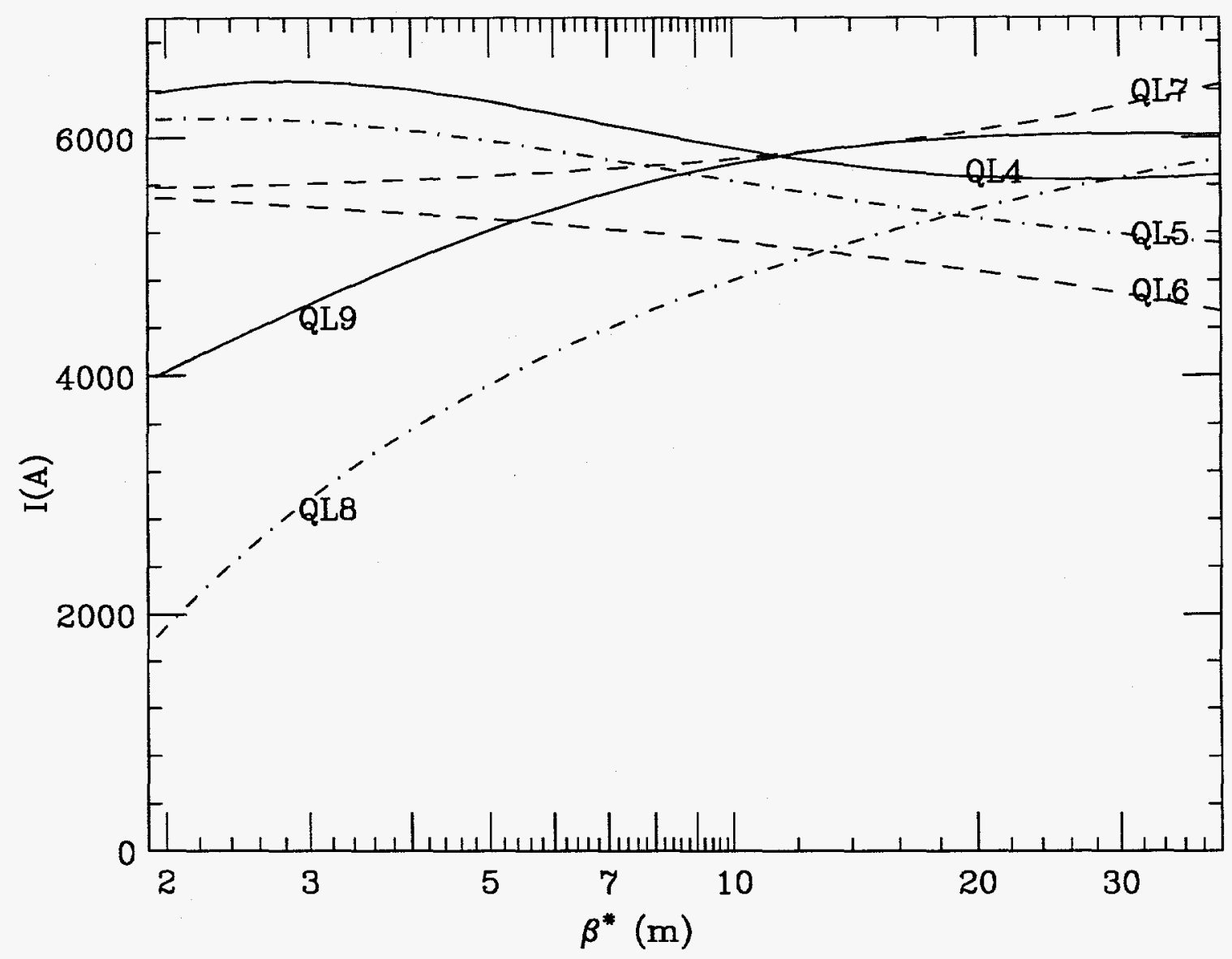

Figure 12: Tuning currents vs $\beta^{*}$ for a medium- $\beta$ IR optics: $L^{*}=90 \mathrm{~m}$. 